\title{
Mean Absolute Input Energy for In-Plane Vibrations of Multiple-Support Structures Subjected to Non-Stationary Horizontal and Rocking Components
}

\author{
M. R. Falamarz-Sheikhabadi ${ }^{1}$, A. Zerva ${ }^{2 *}$ and M. Ghafory-Ashtiany ${ }^{3}$ \\ ${ }^{I}$ Graduate Student, Department of Civil, Architectural and Environmental Engineering, Drexel University, Philadelphia, USA \\ ${ }^{2}$ Professor, Department of Civil, Architectural and Environmental Engineering, Drexel University, Philadelphia, USA \\ ${ }^{3}$ Professor, Department of Special Structures, International Institute of Earthquake Engineering and Seismology, Tehran, Iran \\ ${ }^{*}$ Corresponding Author's Email: zervaa@drexel.edu
}

\begin{abstract}
This study proposes a procedure for the estimation of the mean absolute input energy of multiple-support structures subjected to partially correlated, non-stationary, multi-component, seismic actions. The methodology extends the current evaluation of the earthquake input energy of single-support structures to multiple-support ones, and considers the combined action of translational and rotational components. A mean total velocity response spectrum for characterizing the mean absolute input energy of multiplesupport structures is derived utilizing their pseudo-static response. The earthquake rotational loading is simulated considering the effects of both time delay and loss of coherency. The main advantage of the proposed method is its simplicity of application to a wide range of bridge systems. In addition, the effects of the geometrical configuration of the structure and the earthquake characteristics on the absolute input energy of straight short-span bridges along their longitudinal direction are parametrically investigated, and recommendations for their safe seismic design are proposed. The numerical results of this study indicate that, if the effect of the spatial variation of strong ground motions is incorporated in seismic analysis of bridges, both the differential and the rotational components of the strong ground motions should be taken into account in their excitation.
\end{abstract}

Keywords: Multiple-support structure; Earthquake rotational component; Spatially variable ground motion; Non-stationary excitation; Absolute input energy; Velocity response spectrum; Random vibration analysis.

\section{Introduction}

The characteristics of seismic waves change, both in amplitude and phase, as they travel from the source to the ground surface. The propagation of seismic waves along different paths in the earth media causes spatially varying strong ground motions (SVSGMs) [14]. Past experience regarding strong ground 
motions (SGMs) has shown that, for sites with approximately uniform soil conditions, the differential strong ground motions (DSGMs) at the structure's supports due to SVSGMs may be slightly influential on the seismic excitation of ordinary buildings [27]. However, DSGMs can be detrimental in the seismic behavior of large or extended structures, such as dams [23], and structures on multiple supports, such as bridges $[11,13]$. The contribution of the DSGMs to the structural response of bridges may be considered in their seismic design by modifying conventional seismic analysis methods, such as the response spectrum method [12, 24], and applying asynchronous time series at the structure's supports [28]. Increase of the superstructure rigidity usually increases the DSGM contribution to the seismic response, and leads to structural damage of the piers [39]. Because of this fact, the DSGMs mostly affect the longitudinal response of ordinary straight bridges as compared to their transverse response [56, 57], particularly for bridges with internal expansion joints due to the pounding effects [40]. However, the critical contribution of the DSGMs to the bridge response depends highly on the structural and seismic excitation characteristics, and cannot be easily evaluated before a complete response analysis [29].

The SVSGMs also induce rotational strong ground motions (RSGMs), which can be estimated in terms of the spatial derivatives of their corresponding translational motions [1,2]. These motions, depending on their frequency content, can severely affect the dynamic behavior of structures that are sensitive to highfrequency motions [41], such as nuclear reactors [3, 4], asymmetric buildings [5], slender tower shaped structures [6] or chimneys [41], bridges [7, 8], and vertically irregular structures [9, 10]. The existing records on the RSGMs indicate that they may have large amplitudes close to faults $[15,16]$ and, consequently, can highly contribute to the total response of structures in near-fault zones [17]. The effect of the rotational loading may still remain influential on the structural behavior in the middle-field zone [18], but it should be ignored in far-field regions [34]. The combined action of both the DSGMs and RSGMs on the pseudo-relative spectral velocity (PSV) of a structure on two separate foundations was recently studied using near-fault pulse displacements [58]. The numerical results indicated that, at short periods, the PSV is governed by DSGMs and peak ground velocity, and at long periods, the PSV tends toward an asymptote with amplitude proportional to the maximum rocking angle of the ground motion [58].

Most previous studies on the effects of both DSGMs and RSGMs were performed in the framework of stationary seismic response evaluations $[5,6,12,13]$. However, seismic ground motions are better described by non-stationary random processes, i.e. their intensity and frequency are both time-variant. The dynamic response of structural systems to non-stationary inputs has been addressed in a number of studies [43-47], and the importance of such excitations in evaluating the seismic behavior of structures, for which the fundamental natural period is close to the effective duration of the strong ground motion, 
has been noted [48, 49]. In addition, the appropriate evaluation of the seismic input energy to the systems can only be conducted considering non-stationary inputs due to the dependency of this quantity on the effective duration of the input excitation [59]. Modeling the non-stationarity in the frequency content of the ground motions is a complicated task, and, therefore, most studies have solely considered the nonstationarity in their amplitude to analyze the seismic response of the structures [43-48]. Such an approach may cause limitations on the interpretation of the drift or internal forces of structures [50], but it should not significantly affect the estimation of the input energy to the systems. This is due to the fact that the non-stationarity in the frequency content is related to the phase difference of seismic waves [48], and the earthquake input energy of elastic damped systems does not depend on the phase content of the input motions [51]. Thus, for the evaluation of the input energy of structures, it may be appropriate to represent SGMs as stationary Gaussian random processes modulated by deterministic envelope intensity functions [52].

In the last three decades, even though extensive research efforts have been made to investigate the importance of the DSGMs and RSGMs on the seismic behavior of bridges, their effects have rarely been included in seismic design codes. Eurocode8 (EC8.2) is the only design code that provides provisions for the consideration of the effects of the DSGMs on the seismic response of bridges [25]. This code (EC8.6) also recommends rotational response spectra for considering the RSGMs on the seismic loading of tall structures [42]. However, its provisions could be still improved to fully take into consideration the characteristics of the SVSGMs in the seismic excitations [26,30].

Seismic intensity parameters that are commonly used for medium-to-long period structures are mainly associated with their seismic input energy, such as peak ground velocity, velocity response spectrum and Housner spectrum intensity [38]. These seismic intensities may provide a general insight into the damage induced in bridges based on their dynamic properties, but they cannot consider the effect of the kinematic characteristics of the bridge structure. This issue is addressed herein, and the absolute input energy is considered to establish a platform for the investigation of the SVSGM effect on the seismic behavior of multiple-support structures. The concept of the absolute input energy is commonly accepted as the best representative measure of the seismic input energy of structures, and its importance has been indicated in previous research studies $[19,20]$. In addition, the combined action of the DSGMs and the RSGMs on the non-stationary response of multiple-support structures is investigated. To achieve this, a random vibration procedure is formulated for the estimation of the mean absolute input energy of bridge structures. The SVSGM contribution to the seismic response of straight short-span bridges along their longitudinal direction is parametrically studied, and recommendations for their safe seismic design are proposed. 


\section{Dynamic response of multiple-support structures}

For multiple-support structures, soil-structure interaction (SSI) can also lead to both DSGM and RSGM excitations even under synchronous motions at their supports. However, the present study considers only the kinematic aspect of the seismic loading of multiple-support structures to purely underline the SVSGM contribution to their seismic response. The mathematical formulation for evaluating the dynamic response of multiple-support structures is briefly reviewed in this section. The dynamic equilibrium equation for all degrees-of-freedom (DOFs) of a multiple-support structure can be written in partitioned form, by ignoring SSI, as follows:

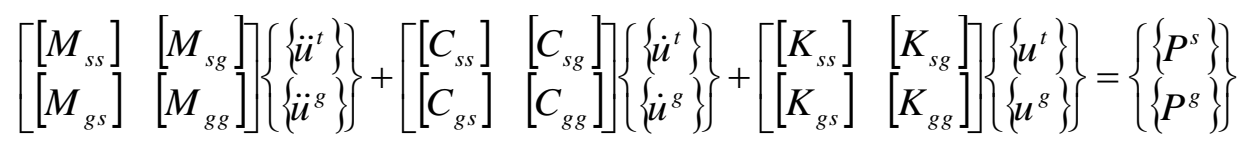

where $[M],[C]$ and $[K]$ are the mass, damping and stiffness matrices, respectively; $\{\ddot{u}\},\{\dot{u}\}$ and $\{u\}$ are the vectors of total accelerations, velocities and displacements, respectively; $\{P\}$ is the force vector; superscripts $g$ and $t$ refer to the ground excitation and the total response of the system, respectively; subscripts $s s$ and $g g$ refer to the structure and ground DOFs, respectively, and subscripts $s g$ and $g s$ refer to the coupling terms between the structure and the ground. By introducing the following matrices:

$\left[r_{M}\right]=-\left[M_{s s}\right]^{-1}\left[M_{s g}\right] \quad, \quad\left[r_{C}\right]=-\left[C_{s s}\right]^{-1}\left[C_{s g}\right] \quad, \quad\left[r_{K}\right]=-\left[K_{s s}\right]^{-1}\left[K_{s g}\right]$

and combining Eqs. 1 and 2, one can obtain:

$\left[M_{s s}\right]\left(\left\{\ddot{u}^{t}\right\}-\left[r_{M}\right]\left\{\ddot{u}^{g}\right\}\right)+\left[C_{s s}\right]\left(\left\{\dot{u}^{t}\right\}-\left[r_{C}\right]\left\{\dot{u}^{g}\right\}\right)+\left[K_{s s}\right]\left(\left\{u^{t}\right\}-\left[r_{K}\right]\left\{u^{g}\right\}\right)=\left\{P^{s}\right\}$

Assuming that:

- there is no mass coupling between the structure and the supports (mass idealization), then, the coupling term $\left\lfloor M_{s g}\right\rfloor$ is zero, and $\left[r_{M}\right]=[0]$;

- the system is linear with stiffness-proportional damping, i.e. $[r]=\left[r_{K}\right]=\left[r_{C}\right]$, and

- the excitation of the system is caused only by the SGMs at the foundations, and, hence, $\left\{P^{s}\right\}=\{0\}$,

Eq. 3 can be simplified to:

$\left[M_{s s}\right]\left\{\ddot{u}^{d}\right\}+\left[C_{s s}\right]\left\{\dot{u}^{d}\right\}+\left[K_{s s}\right]\left\{u^{d}\right\}=-\left[M_{s s}\right]\left\{\ddot{u}^{p s}\right\}$ 
where $\left\{\ddot{u}^{p s}\right\}=[r]\left\{\ddot{u}^{g}\right\}$ and $\left\{\ddot{u}^{d}\right\}=\left\{\ddot{u}^{t}\right\}-[r]\left\{\ddot{u}^{g}\right\}$ are, respectively, the pseudo-static and dynamic acceleration vector of the structure. For a system with $N$ DOFs and diagonal mass matrix, the dynamic velocity response of the $k^{\text {th }}$ DOF can be expressed as:

$\dot{u}_{k}^{d}(t)=-\sum_{j=1}^{N} \sum_{i=1}^{N} \phi_{k j} \Gamma_{i j} \int_{0}^{t} h_{V j}(t-\tau) \ddot{u}_{i}^{p s}(\tau) d \tau$

where $\phi_{k j}$ is the value of the $j^{\text {th }}$ mode shape at the $k^{\text {th }} \mathrm{DOF}$, and $\Gamma_{i j}$ and $h_{V j}$ are defined as follows:

$$
\begin{aligned}
& \Gamma_{i j}=\frac{\phi_{i j} m_{i}}{M_{j}} \\
& h_{V j}(t)=\left[\frac{\xi_{j} \omega_{j}}{\omega_{d j}} \sin \left(\omega_{d j} t\right)-\cos \left(\omega_{d j} t\right)\right] \exp \left[-\xi_{j} \omega_{j} t\right]
\end{aligned}
$$

in which $m_{i}$ is the mass of the $i^{\text {th }} \mathrm{DOF}, M_{j}$ is the $j^{\text {th }}$ modal mass, and $\omega_{j}, \omega_{d j}$ and $\xi_{j}$ are the natural frequency, damped frequency, and viscous damping ratio for the $j^{\text {th }}$ mode, respectively. Considering the ground motion at the $l^{\text {th }}$ support of the structure, $\ddot{u}_{l}^{g}(t)$, as a non-stationary excitation defined by the product of a deterministic modulating function, $f(t)$, and a zero-mean stationary random process, $\ddot{x}_{l}^{g}(t)$, one can obtain:

$\ddot{u}_{i}^{p s}(t)=\left[r_{i}\right]\left\{\ddot{u}^{g}(t)\right\}=\sum_{l=1}^{N_{g}} r_{i l} \ddot{u}_{l}^{g}(t)=\sum_{l=1}^{N_{g}} r_{i l} f(t) \ddot{x}_{l}^{g}(t)=f(t)\left[r_{i}\right]\left\{\ddot{x}^{g}(t)\right\}=f(t) a_{i}^{p s}(t)$

where $\left[r_{i}\right]$ refers to the $i^{\text {th }}$ row of the matrix $[r], N_{g}$ indicates the number of the structural supports, and $a_{i}^{p s}(t)=\left[r_{i}\right]\left\{\ddot{x}^{g}(t)\right\}$. Substituting Eq. 7 into Eq. 5 results in:

$$
\begin{aligned}
\dot{u}_{k}^{d}(t) & =-\sum_{j=1}^{N} \sum_{i=1}^{N} \phi_{k j} \Gamma_{i j} \int_{0}^{t} h_{V j}(t-\tau) f(\tau) a_{i}^{p s}(\tau) d \tau=-\sum_{j=1}^{N} \sum_{i=1}^{N} \phi_{k j} \Gamma_{i j} \int_{0}^{t} h_{V j}(t-\tau) f(\tau)\left(\int_{-\infty}^{+\infty} A_{i}^{p s}(\omega) e^{i \omega \tau} d \omega\right) d \tau \\
& =-\sum_{j=1}^{N} \sum_{i=1}^{N} \phi_{k j} \Gamma_{i j} \int_{-\infty}^{+\infty} M_{j}^{*}(t, \omega) A_{i}^{p s}(\omega) e^{i \omega t} d \omega
\end{aligned}
$$

where $A_{i}^{p s}(\omega)$ is the Fourier transform of $a_{i}^{p s}(\tau)$, and:

$$
M_{j}^{*}(t, \omega)=\int_{0}^{t} h_{V j}(t-\tau) f(\tau) e^{-i \omega(t-\tau)} d \tau=e^{-i \omega t} \int_{0}^{t} h_{V j}(t-\tau) f(\tau) e^{i \omega \tau} d \tau
$$


$M_{j}^{*}(t, \omega)$ may be interpreted as the product of $e^{-i \omega t}$ and the velocity response of a single DOF oscillator to an input excitation $f(t) e^{i \omega t}$. The complex conjugate of this function is utilized in Section 3 to evaluate the mean absolute input energy of multiple-support structures. To obtain the mean-square dynamic response of the considered $N$ DOFs multiple-support system, it is required to derive the entries of the spectral density function (SDF) matrix corresponding to the pseudo-static accelerations (Eq. 7):

$S_{i_{i} i_{k}}^{p s}(t, \omega)=f^{2}(t)\left[r_{i}\right]\left[S_{\ddot{x} x}^{g g}(\omega)\right]\left[r_{k}\right]^{T}$

where:

$\left[S_{\ddot{x} \ddot{x}}^{g g}(\omega)\right]=\left[\begin{array}{ccccc}1 & \gamma_{12} & \cdot & \cdot & \gamma_{1 N} \\ \gamma_{21} & \cdot & & & \cdot \\ \cdot & & \cdot & & \cdot \\ \cdot & & & \cdot & \cdot \\ \gamma_{N 1} & \cdot & \cdot & \cdot & 1\end{array}\right] S_{\ddot{x \ddot{x}}}^{g}(\omega)$

$\gamma_{l p}$ is the coherency function of the motions at locations $l$ and $p$ on the ground surface, and $S_{\ddot{x} \dot{g}}^{g}(\omega)$ is the SDF of $\ddot{x}_{g}(t)$. If the entries of $\left[S_{\ddot{x} \ddot{x}}^{g g}(\omega)\right]$ are expressed as:

$S_{\ddot{x}_{l} \ddot{x}_{p}}^{g g}\left(x_{l}, x_{p}, \omega\right)=\gamma_{l p}\left(x_{l}, x_{p}, \omega\right) S_{\ddot{x} \ddot{x}}^{g}(\omega)$

Eq. 10 can be simplified to:

$S_{\ddot{u}_{i} i_{k}}^{p s}(t, \omega)=f^{2}(t) S_{\dot{x} \ddot{x}}^{g}(\omega) \sum_{l=1}^{N_{g}} \sum_{p=1}^{N_{g}} r_{i l} \gamma_{l p}\left(x_{l}, x_{p}, \omega\right) r_{k p}$

Herein, the following one-dimensional functional form for the coherency function is considered to model the DSGM effects [22]:

$\gamma_{l p}\left(x_{l}, x_{p}, \omega\right)=\mid \gamma_{l p}\left(x_{l}, x_{p}, \omega\right) \exp \left[-i \omega \frac{x_{l}-x_{p}}{V_{x}}\right]=\exp \left[-\left(\frac{\lambda_{x} \omega\left(x_{l}-x_{p}\right)}{V_{S}}\right)^{2}\right] \exp \left[-i \omega \frac{x_{l}-x_{p}}{V_{x}}\right]$

in which $\left|\gamma_{l p}\left(x_{l}, x_{p}, \omega\right)\right|$ is the lagged coherency (loss) function, $\lambda_{x}$ is a dimensionless factor with values typically in the range between 0 and $0.5, V_{S}$ is the shear wave velocity in the random medium, and $V_{x}$ is the constant apparent velocity of the seismic waves along the $x$-axis of the Cartesian coordinate system, which, in the present analysis, coincides with the longitudinal direction of the bridge (Fig. 1). Fig. 2 presents a schematic diagram of the wave propagation mechanism of a seismic event from its hypocenter 
to the site. For the investigation of the wave propagation effects, the apparent velocity is considered as $V_{S} / \sin \beta$, in which $\beta$ is the incident angle to the vertical line in the $x$-z plane (Fig. 2).

The formulation presented in this section is valid for both the rocking and the horizontal component excitation by taking into consideration that the entries of the matrix $[r]$ are different for these excitations. In the following, the pseudo-static input of a typical straight short-span bridge supported on $N_{g}$ isolated columns with the following characteristics is evaluated:

- The bridge deck can be considered rigid in its own plane, as, e.g. a bridge with a concrete box-girder deck with its length to width ratio being less than eight [32], and

- The mass of the bents is negligible compared to that of the deck, i.e. one massless element is used to model each column. This assumption has generally been accepted for low-to-medium height bridges and can be partially modified if equivalent masses due to the bents need to be added to the superstructure mass [33].

The pseudo-static acceleration of the superstructure of such a bridge due to the earthquake horizontal and rocking components can be obtained as:

$$
\left\{\ddot{u}^{p s}(t)\right\}^{T}=\left\{\frac{1}{\sum_{l=1}^{N_{g}} K_{x l}} \sum_{l=1}^{N_{g}} K_{x l}\left[\ddot{u}_{x l}^{g}+h_{l} \ddot{\theta}_{y l}^{g}\right], \quad \frac{1}{\sum_{l=1}^{N_{g}} K_{y l}} \sum_{l=1}^{N_{g}} K_{y l}\left[\ddot{u}_{y l}^{g}+h_{l} \ddot{\theta}_{x l}^{g}\right], \quad \frac{1}{\sum_{l=1}^{N_{g}} K_{y l} x_{l}^{2}} \sum_{l=1}^{N_{g}} x_{l} K_{y l}\left[\ddot{u}_{y l}^{g}+h_{l} \ddot{\theta}_{x l}^{g}\right]\right\}
$$

in which $x_{l}$ denotes the coordinate of the $l^{\text {th }}$ column with stiffness $K_{i l}(i=x$ or $y$ ) with respect to the superstructure's stiffness center, $h_{l}$ is the height of the $l^{\text {th }}$ column, and $\ddot{\theta}_{i l}^{g} \quad(i=x$ or $y$ ) is the rocking acceleration component about the $i$-axis at the base of the $l^{\text {th }}$ column. The first and second entries of the vector of the pseudo-static acceleration of the superstructure are, respectively, the translational acceleration components along the $x$ - and $y$-direction of the superstructure, and the third entry is the torsional acceleration component (rotation about the $z$-axis) of the superstructure (Fig. 1). It is further noted that the first terms in the bracket of the first and second entries represent the translational ground response, whereas the second terms in the brackets the translational response caused by the rocking ground excitation. Assuming that all bridge supports have approximately the same height $\left(h_{l}=h\right)$ and lateral stiffness, the diagonal entries of the SDFs of the pseudo-static acceleration of the bridge deck can be expressed as: 


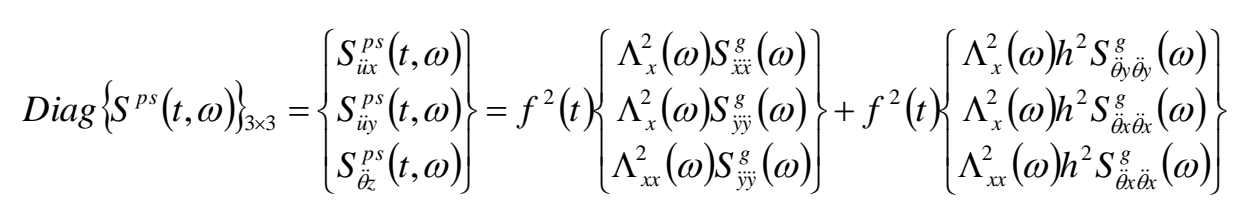

where:

$$
\begin{aligned}
& \Lambda_{x}^{2}(\omega)=\frac{1}{N_{g}^{2}}\left[N_{g}+2 \sum_{p=1}^{N_{g}-1} \sum_{l=p+1}^{N_{g}} \operatorname{Re}\left[\gamma_{l p}\left(x_{l}, x_{p}, \omega\right)\right]\right] \\
& \Lambda_{x x}^{2}(\omega)=\frac{1}{\left(\sum_{l=1}^{N_{g}} x_{l}^{2}\right)^{2}}\left[\sum_{l=1}^{N_{g}} x_{l}^{2}+2 \sum_{p=1}^{N_{g}-1} \sum_{l=p+1}^{N_{g}} x_{l} x_{p} \operatorname{Re}\left[\gamma_{l p}\left(x_{l}, x_{p}, \omega\right)\right]\right]
\end{aligned}
$$

in which Re[.] indicates the real part of the quantity in the brackets. In a special case, when adjacent supports are at equal distances from each other and the seismic waves propagate on the ground surface along a direction that forms an angle $\alpha(0 \leq \alpha \leq \pi / 2)$ with the longitudinal axis of the bridge $(x$ direction), the following basic relations for $\Lambda_{x}(\omega)$ and $\Lambda_{x x}(\omega)$ can be derived using Eq. 14 and some algebraic manipulations:

$$
\begin{aligned}
& \Lambda_{x}^{2}(\omega)=\frac{1}{N_{g}^{2}}\left[N_{g}+2 \sum_{l=1}^{N_{g}-1}\left\{\left(N_{g}-l\right) \exp \left[-\left(\frac{l \lambda_{x} \eta}{N_{g}-1}\right)^{2}\right] \cos \left(\frac{l \eta}{N_{g}-1} \sin \beta\right)\right\}\right] \\
& \Lambda_{x x}^{2}(\omega)=\frac{1}{b_{N_{g}}^{2} d^{2}}\left[b_{N_{g}}+2 \sum_{l=1}^{N_{g}-1}\left\{a_{l} \exp \left[-\left(\frac{l \lambda_{x} \eta}{N_{g}-1}\right)^{2}\right] \cos \left(\frac{l \eta}{N_{g}-1} \sin \beta\right)\right\}\right]
\end{aligned}
$$

where $\eta=\omega d \cos \alpha / V_{S}, d$ is the distance between the first and the last pier of the bridge (Fig. 1), and the values of $b_{N g}$ and $a_{l}$ are provided in Table 1 for a practical range of continuous bridges with relatively rigid decks $\left(2 \leq N_{g} \leq 8\right)$. Obviously, if seismic waves propagate along the $x$-direction, then $\eta=\omega d / V_{S}$.

\section{Mean absolute input energy of multiple-support structures}

Herein, a new relation for the evaluation of the mean absolute input energy of multiple-support structures is presented, and its application to straight short-span bridges, with the consideration of the two conditions specified in Section 2, is discussed. The formulation can be used for both rocking and horizontal components, with the only differences between these two cases being the entries of the matrix 
[r]. The concept of the estimation of the absolute input energy of single-support structures [19], with $N$ DOFs, can be extended to multiple-support structures as:

$$
W_{I}^{t o t}=\int_{0}^{t}\left\{\dot{u}^{p s}(t)\right\}_{1 \times N}^{T}\left[M_{s s}\right]_{N \times N}\left\{\ddot{u}^{t}(t)\right\}_{N \times 1} d t
$$

For structures with diagonal mass matrix, Eq. 19 can be expressed as:

$$
W_{I}^{t o t}(t)=\int_{0}^{t} \sum_{i=1}^{N} \ddot{u}_{i}^{t} m_{i} \dot{u}_{i}^{p s} d t=\int_{0}^{t} \sum_{i=1}^{N} \ddot{u}_{i}^{t} m_{i}\left(\dot{u}_{i}^{t}-\dot{u}_{i}^{d}\right) d t=\int_{0}^{t} \sum_{i=1}^{N} \ddot{u}_{i}^{t} m_{i} \dot{u}_{i}^{t} d t-\int_{0}^{t} \sum_{i=1}^{N}\left(\ddot{u}_{i}^{d}+\ddot{u}_{i}^{p s}\right) m_{i} \dot{u}_{i}^{d} d t
$$

Noting that the relative input energy of the structure subjected to the seismic action is $W_{I}(t)=-\sum_{i=1}^{N} m_{i} \int_{0}^{t} \dot{u}_{i}^{d}(t) \ddot{u}_{i}^{p s}(t) d t$, the relationship between the relative and absolute input energy becomes:

$$
\begin{aligned}
W_{I}^{\text {tot }}(t) & =\sum_{i=1}^{N}\left(\int_{0}^{t} m_{i} \ddot{u}_{i}^{t} \dot{u}_{i}^{t} d t-\int_{0}^{t} m_{i} \ddot{u}_{i}^{d} \dot{u}_{i}^{d} d t-\int_{0}^{t} m_{i} \dot{u}_{i}^{d} \ddot{u}_{i}^{p s} d t\right)=\sum_{i=1}^{N}\left(\frac{1}{2} m_{i}\left(\dot{u}_{i}^{t}\right)^{2}-\frac{1}{2} m_{i}\left(\dot{u}_{i}^{d}\right)^{2}\right)+W_{I}(t) \rightarrow \\
W_{I}^{\text {tot }}(t) & =\sum_{i=1}^{N} \frac{1}{2} m_{i}\left(\dot{u}_{i}^{p s}\right)^{2}+\sum_{i=1}^{N} m_{i} \dot{u}_{i}^{d} \dot{u}_{i}^{p s}+W_{I}(t)
\end{aligned}
$$

In the following, the expected value, $E[$.$] , of each term on the right-hand side of Eq. 21$ is evaluated. In this regard, $\dot{u}_{i}^{p s}(t)$ is defined as:

$$
\dot{u}_{i}^{p s}(t)=\left[r_{i}\right]\left\{\dot{u}^{g}(t)\right\}=\sum_{l=1}^{N_{g}} r_{i l} \dot{u}_{l}^{g}(t)=\sum_{l=1}^{N_{g}} r_{i l} f(t) \dot{x}_{l}^{g}(t)=f(t)\left[r_{i}\right]\left\{\dot{x}^{g}(t)\right\}=f(t) v_{i}^{p s}(t)
$$

Considering $f(t)$ as a box-car type modulating function:

$$
f(t)=\left\{\begin{array}{ccc}
f_{0} & \text { for } & 0 \leq t \leq t_{d} \\
0 & \text { for } & t>t_{d}
\end{array}\right.
$$

where $f_{0}$ is the scaling factor, and $t_{d}$ is the effective duration of the seismic input, it can be shown that:

$$
\dot{u}_{l}^{g}(t)=f(t) \dot{x}_{l}^{g}(t) \rightarrow \ddot{u}_{l}^{g}(t)=f(t) \ddot{x}_{l}^{g}(t)
$$

Thus, the use of Eq. 7 to define $\ddot{u}_{i}^{p s}(t)$ is justified. The expected value of the first term on the right-hand side of Eq. 21, using Eqs. 13 and 22, can be expressed as: 


$$
\begin{aligned}
& E\left[\sum_{i=1}^{N} \frac{1}{2} m_{i} \dot{u}_{i}^{p s}(t) \dot{u}_{i}^{p s}(t)\right]=\sum_{i=1}^{N} \frac{1}{2} m_{i} f^{2}(t) R_{v_{i} v_{i}}^{p s}(t) \\
& =\sum_{i=1}^{N} \frac{1}{2} m_{i} f^{2}(t) \int_{-\infty}^{+\infty} S_{v_{i} v_{i}}^{p s}(\omega) d \omega=\sum_{i=1}^{N} \sum_{p=1}^{N_{g}} \sum_{l=1}^{N_{g}} \frac{1}{2} r_{i l} r_{i p} m_{i} f^{2}(t) \int_{-\infty}^{+\infty} \gamma_{l p}\left(x_{l}, x_{p}, \omega\right) S_{\dot{x} \dot{x}}^{g}(\omega) d \omega
\end{aligned}
$$

where $R(t)$ is the autocorrelation function. The expected value of the second term on the right-hand side of Eq. 21, using Eqs. 5, 7, 13 and 22, can be evaluated as:

$$
\begin{aligned}
& E\left[\sum_{i=1}^{N} m_{i} \dot{u}_{i}^{d}(t) \dot{u}_{i}^{p s}(t)\right]=E\left[\sum_{i=1}^{N} \sum_{k=1}^{N} \sum_{j=1}^{N} m_{i} \varphi_{i j} \Gamma_{k j} \int_{0}^{t} \ddot{u}_{k}^{p s}(\tau) \dot{u}_{i}^{p s}(t) h_{V j}(t-\tau) d \tau\right] \\
& =\sum_{i=1}^{N} \sum_{k=1}^{N} \sum_{j=1}^{N} \sum_{p=1}^{N_{g}} \sum_{l=1}^{N_{g}} r_{k l} r_{i p} m_{i} \varphi_{i j} \Gamma_{k j} f(t) \int_{0}^{t} h_{V j}(t-\tau) f(\tau) \int_{-\infty}^{+\infty} \gamma_{l p}\left(x_{l}, x_{p}, \omega\right) S_{\dot{x} \dot{g}}^{g}(\omega) e^{i \omega(t-\tau)} d \omega d \tau \\
& =\sum_{i=1}^{N} \sum_{k=1}^{N} \sum_{j=1}^{N} \sum_{p=1}^{N_{g}} \sum_{l=1}^{N_{g}} r_{k l} r_{i p} m_{i} \varphi_{i j} \Gamma_{k j} f(t) \int_{-\infty}^{+\infty} \gamma_{l p}\left(x_{l}, x_{p}, \omega\right) M_{j}(t, \omega) S_{\dot{i x}}^{g}(\omega) d \omega
\end{aligned}
$$

where $M_{j}(t, \omega)$ is the complex conjugate of $M_{j}^{*}(t, \omega)$ (Eq. 9 in Section 2). The expected value of the last term in the right-hand side of Eq. 21, using Eqs. 5, 7 and 13, can also be obtained as:

$$
\begin{aligned}
& E\left[W_{I}(t)\right]=E\left[-\sum_{i=1}^{N} m_{i} \int_{0}^{t} \dot{u}_{i}^{d}(t) \ddot{u}_{i}^{p s}(t) d t\right]=-E\left[\sum_{i=1}^{N} \sum_{k=1}^{N} \sum_{j=1}^{N} m_{i} \varphi_{i j} \Gamma_{k j} \int_{0}^{t} \int_{0}^{t} \ddot{u}_{k}^{p s}(\tau) \ddot{u}_{i}^{p s}(t) h_{V j}(t-\tau) d \tau d t\right] \\
& =-\sum_{i=1}^{N} \sum_{k=1}^{N} \sum_{j=1}^{N} \sum_{p=1}^{N_{g}} \sum_{l=1}^{N_{g}} r_{k l} r_{i p} m_{i} \varphi_{i j} \Gamma_{k j} \int_{0}^{t} \int_{0}^{t} h_{V j}(t-\tau) f(\tau) f(t) \int_{-\infty}^{+\infty} \gamma_{l p}\left(x_{l}, x_{p}, \omega\right) S_{\ddot{x}}^{g}(\omega) e^{i \omega(t-\tau)} d \omega d \tau d t \\
& =-\sum_{i=1}^{N} \sum_{k=1}^{N} \sum_{j=1}^{N} \sum_{p=1}^{N_{g}} \sum_{l=1}^{N_{g}} r_{k l} r_{i p} m_{i} \varphi_{i j} \Gamma_{k j}^{+\infty} \int_{-\infty}^{+\infty} \gamma_{l p}\left(x_{l}, x_{p}, \omega\right) N_{j}(t, \omega) S_{\ddot{x} \dot{g}}^{g}(\omega) d \omega
\end{aligned}
$$

where:

$$
N_{j}(t, \omega)=\int_{0}^{t} \int_{0}^{t} h_{V j}(t-\tau) f(\tau) f(t) e^{i \omega(t-\tau)} d \tau d t=\int_{0}^{t} M_{j}(t, \omega) f(t) d t
$$

$M_{j}(t, \omega)$ and $N_{j}(t, \omega)$ for a box-car type modulating function are evaluated in Appendix A. The mean absolute input energy of multiple-support structures can then be evaluated by the summation of Eqs. 2527. For straight short-span bridges, with the consideration of the two conditions specified in Section 2, subjected to the horizontal, $\ddot{u}_{x}^{g}(t)$, and rocking, $\ddot{\theta}_{y}^{g}(t)$, components of the earthquake, the mean absolute input energy can be obtained as: 


$$
\begin{aligned}
& E\left[W_{u I}^{t o t}(t)\right]=\frac{1}{2} m f^{2}(t) \int_{-\infty}^{+\infty} \frac{\Lambda_{x}^{2}(\omega)}{\omega^{2}} S_{\ddot{x} x}^{g}(\omega) d \omega+m \int_{-\infty}^{+\infty} \Lambda_{x}^{2}(\omega) S_{\ddot{x} x}^{g}(\omega)\left[\frac{f(t)}{i \omega} M(t, \omega)-N(t, \omega)\right] d \omega
\end{aligned}
$$

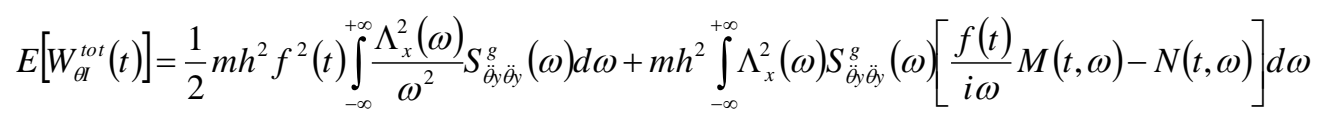

where subscripts $u$ and $\theta$ refer to the horizontal and rocking component excitation, respectively. $M(t, \omega)$ and $N(t, \omega)$ can be written as:

$$
\begin{aligned}
& M(t, \omega)=\operatorname{Re}[M(t, \omega)]+i \operatorname{Im}[M(t, \omega)] \\
& N(t, \omega)=\operatorname{Re}[N(t, \omega)]+i \operatorname{Im}[N(t, \omega)]
\end{aligned}
$$

Considering the fact that $\Lambda_{x}^{2}(\omega), S_{\ddot{x} \ddot{x}}^{g}(\omega)$ and $S_{\ddot{\ddot{y} y} \ddot{y} y}^{g}(\omega)$ are even functions, and with the properties of the functions $M(t, \omega)$ and $N(t, \omega)$ as provided in Appendix A, Eq. 29 can be simplified to:

$$
\begin{aligned}
& E\left[W_{u I}^{t o t}(t)\right]=m f^{2}(t) \int_{0}^{+\infty} \frac{\Lambda_{x}^{2}(\omega)}{\omega^{2}} S_{\ddot{x} x}^{g}(\omega) d \omega+2 m \int_{0}^{+\infty} \Lambda_{x}^{2}(\omega) S_{\ddot{x x}}^{g}(\omega)\left[\frac{f(t)}{\omega} \operatorname{Im}[M(t, \omega)]-\operatorname{Re}[N(t, \omega)]\right] d \omega \\
& E\left[W_{\theta l}^{t o t}(t)\right]=m h^{2} f^{2}(t) \int_{0}^{+\infty} \frac{\Lambda_{x}^{2}(\omega)}{\omega^{2}} S_{\ddot{\partial y} \ddot{y} y}^{g}(\omega) d \omega+2 m h^{2} \int_{0}^{+\infty} \Lambda_{x}^{2}(\omega) S_{\ddot{\partial} y \ddot{y}}^{g}(\omega)\left[\frac{f(t)}{\omega} \operatorname{Im}[M(t, \omega)]-\operatorname{Re}[N(t, \omega)]\right] d \omega
\end{aligned}
$$

In the following section, the combined action of the RSGM and DSGM on the absolute input energy of straight short-span bridges is studied by introducing the mean total velocity response spectrum (MTVRS) as:

$\operatorname{MTVRS}^{u}=\sqrt{\frac{\max \left\{E\left[W_{u I}^{\text {tot }}(t)\right]\right\}}{m / 2}}, \quad \operatorname{MTVRS}^{u, \theta}=\sqrt{\frac{\max \left\{E\left[W_{u I}^{\text {tot }}(t)\right]\right\}+\max \left\{E\left[W_{\theta I}^{\text {tot }}(t)\right]\right\}}{m / 2}}$

where $m$ is set equal to unity. Herein, the assumption of a unit mass for the superstructure is utilized in order to investigate the characteristics of the proposed spectrum, and to generalize the trend of the numerical results to similar bridges but with different deck weight. The following ratios are also defined for the investigation of the contribution of the various types of input excitations:

$$
\tau_{S V}^{1}=\frac{\operatorname{MTVRS}_{\text {nonuniform }}^{u}}{\operatorname{MTVRS}_{\text {uniform }}^{u}}, \quad \tau_{S V}^{2}=\frac{\operatorname{MTVRS}_{\text {nonuniform }}^{u, \theta}}{\operatorname{MTVRS}_{\text {uniform }}^{u}}, \quad \tau_{S V}^{3}=\frac{\operatorname{MTVRS}_{\text {nonuniform }}^{u, \theta}}{\operatorname{MTVRS}_{\text {nonuniform }}^{u}}
$$

where subscript $S V$ refers to spectral velocity, and subscripts uniform and nonuniform indicate that the MTVRS is evaluated by considering either the uniform or the non-uniform seismic excitation at the bridge supports. 


\section{Numerical results}

In this section, the response of straight, short-span bridges, with the consideration of the two conditions specified in Section 2, subjected to non-stationary, multi-support, multi-component excitations is parametrically studied. The characteristics of the input motion are reviewed in Section 4.1, and the response of the bridges is discussed using the MTVRS in Section 4.2.

\subsection{Input excitation}

Both time delay and loss of coherency effects should be incorporated in the evaluation of the earthquake rotational components. The SDF of the rocking component corresponding to a point on the ground surface may be considered equivalent to the SDF of the slope of the line that connects two points when their separation distance approaches zero [31]:

$$
S_{\ddot{\partial y} \ddot{\theta}_{y}}^{g}=\lim _{x \rightarrow 0} \frac{2 S_{\ddot{z} z}^{g}(\omega)}{x^{2}}\left[1-\left|\gamma_{l p}(0, x, \omega)\right| \cos \left(\omega x / V_{x}\right)\right]
$$

where $S_{\ddot{z} \check{z}}^{g}(\omega)$ is the SDF of the vertical acceleration component. The solution of the above equation can be obtained using L'Hopital's rule twice as follows [31]:

$$
S_{\ddot{\partial} y \ddot{y},}^{g}(\omega)=2 \omega^{2} S_{\ddot{z} z}^{g}(\omega)\left[\frac{\lambda_{x}^{2}}{V_{S}^{2}}+\frac{1}{2 V_{x}^{2}}\right]
$$

The functional form and parameters of coherency models for the SVSGMs are usually derived by fitting the model to coherency estimates of data recorded at dense instrument arrays. Hence, the use of a point model for the estimation of the rocking SDF, when $x$ tends to zero, should be applied with caution. From a practical viewpoint, the accuracy of such an approach can be improved by evaluating the parameters of the considered coherency function for separation distances comparable to the foundation dimensions of structures, or considering coherency functions fitted to data recorded at short separation distances. Such dense instrument arrays have been deployed around the world and the coherency of their data evaluated as, e.g. the LSST array with minimum station separation distance of $6 m$ [60]. It is noted, however, that the coherency model selected in this study (Eq. 14) is semi-analytical, as it is based on wave propagation through random media [22], and, therefore, its use in the estimation of the rocking SDF is justified. 
The angular displacement and velocity response spectrum resulting from the rocking component of the excitation can be obtained using:

$$
S D^{2}=C_{d}^{2} \int_{-\infty}^{+\infty} S^{g}(\omega)\left|H_{D}(\omega)^{2} d \omega \quad, \quad S V^{2}=C_{v}^{2} \int_{-\infty}^{+\infty} \omega^{2} S^{g}(\omega)\right| H_{D}(\omega)^{2} d \omega
$$

where $S D$ and $S V$ are the displacement and velocity response spectra, respectively, $C_{d}$ and $C_{v}$ are their corresponding peak factors, and $H_{D}(\omega)$ is the displacement transfer function:

$$
H_{D}(\omega)=\frac{1}{m\left[\left(\omega_{1}^{2}-\omega^{2}\right)+2 i \xi_{1} \omega_{1} \omega\right]}
$$

where $\omega_{1}$ and $\xi_{1}$ are the fundamental natural frequency and damping ratio of a single DOF oscillator, respectively. Substituting Eq. 35 into Eq. 36 results in:

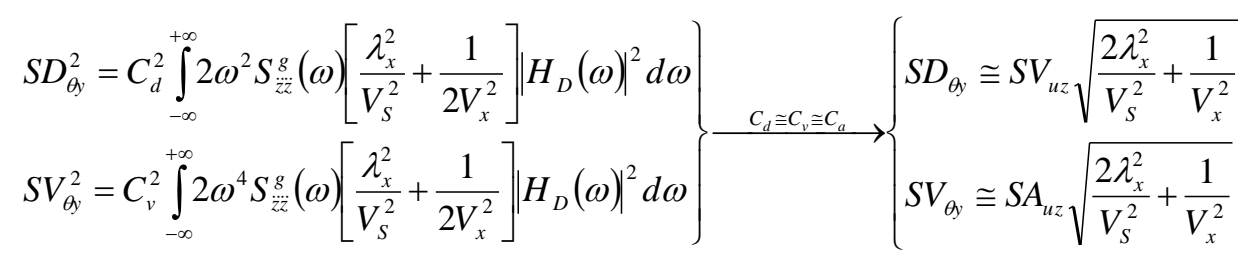

where $S A$ is the acceleration response spectrum, and $C_{a}$ its corresponding peak factor. In random vibration, the Kanai-Tajimi spectrum [36, 37] is commonly used to model seismic excitations. The main feature of the Kanai-Tajimi spectrum, apart from its simplicity, is that it accounts for the resonant filtering effect that the surface soil deposit has on propagating seismic waves, i.e. a clear physical interpretation associated with site specific soil conditions. However, this spectrum has an unrealistic non-zero value at $\omega=0$ and a high-pass filter should be utilized to compensate this problem [21]. In addition, for large $\omega$, the rocking spectrum (Eq. 35) diverges, its root-mean-square becomes infinite and, thus, a cut-off frequency [35] or a low-pass filter should be adopted [31]. Herein, to model the SDF of the input translational acceleration, a double-filtered Kanai-Tajimi spectrum is defined as follows:

$$
\frac{S^{g}(\omega)}{S_{0}}=\frac{\left(\omega / \omega_{f}\right)^{4}}{\left[1-\left(\omega / \omega_{f}\right)^{2}\right]^{2}+4 \xi_{f}\left(\omega / \omega_{f}\right)^{2}} \times \frac{1+4 \xi_{g}^{2}\left(\omega / \omega_{g}\right)^{2}}{\left[1-\left(\omega / \omega_{g}\right)^{2}\right]^{2}+4 \xi_{g}^{2}\left(\omega / \omega_{g}\right)^{2}} \times \exp \left(-\frac{\left(\omega / \omega_{g}\right)^{2}}{2\left(1+\xi_{g}\right)^{2}}\right)
$$

where $\omega_{g}, \omega_{f}, \xi_{g}, \xi_{f}$ and $S_{0}$ are empirical parameters determined by fitting Eq. 39 to the SDF of recorded accelerograms. The last term in the right-hand side of Eq. 39 is a low-pass exponential filter that rapidly decreases the amplitude of the spectrum after its upper half-power point, i.e. $\omega_{g}\left(1+\xi_{g}\right)$. Hereafter, the single-filtered SDF refers to the Kanai-Tajimi spectrum with only one high-pass filter as proposed in Ref. [21], and the double-filtered SDF indicates the Kanai-Tajimi spectrum with both high- 
and low-pass filters (Eq. 39). Table 2 provides typical values of the Kanai-Tajimi spectrum corresponding to a medium soil [12].

Fig. 3 presents the SDF of the translational and rocking acceleration components for the single- and double-filtered Kanai-Tajimi spectrum with the parameters provided in Table 2. The rocking SDF is estimated using Eq. 35 and considering $V_{S}=300 \mathrm{~m} / \mathrm{sec}$ and $\beta=30^{\circ}$. Table 3 provides the predominant periods of the acceleration, velocity and displacement SDFs of the horizontal and rocking components, obtained from the frequencies corresponding to the peak values of the spectra. Two values are considered for $\lambda_{x}$ to study the effect of the loss of coherency on the amplitude of the SDF of the rocking component: (1) $\lambda_{x}=0$, i.e. only time delay effects, and (2) $\lambda_{x}=0.3$, i.e. both time delay and loss of coherency effects are considered in the estimation of the rocking component. As can be seen from Figs. 3(a) and (c), there is no significant difference between the SDF of the horizontal and vertical acceleration components obtained from the single- and double-filtered Kanai-Tajimi spectrum. However, this is not the case for the SDF of the rocking acceleration component obtained from the single- and double-filtered Kanai-Tajimi spectrum. Figs. 3(b) and (d) indicate that, at low frequencies, both spectra have the same shape, but at high frequencies, only the amplitude of the double-filtered Kanai-Tajimi spectrum decreases after its upper half-power point. This comparison suggests the fairly good capacity of the double-filtered KanaiTajimi spectrum (Eq. 39) in filtering the high-frequency parts of the rocking SDF. From Eq. 35 and Fig. 3(d) it is obvious that the consideration of the loss of coherency increases the amplitude of the SDF of the rocking component, and, consequently, leads to a more critical rotational seismic loading. Therefore, it is not conservative to omit the effect of the loss of coherency in the evaluation of the rotational loading in structural analyses.

The non-stationary excitation of a system depends on the effective duration of the SGM through its modulating intensity function $[53,54]$. Herein, the effective duration of the SGM is evaluated based on the Modified Mercalli intensity, $I_{M M}$, as [55]:

$$
t_{d}=\left\{\begin{array}{lll}
46.5-3.85 I_{M M} & \text { for } & \text { horizontal acceleration component } \\
56.3-4.67 I_{M M} & \text { for } & \text { vertical acceleration component }
\end{array}\right.
$$

where $t_{d}$ is measured in seconds, and $I I I \leq I_{M M} \leq V I I I$. Considering that the rocking component is derived from the vertical component, its duration is estimated based on that of the vertical component. In addition, the value of the scale factor, $f_{0}$, can be obtained by considering the mean Arias intensity as [53]:

$$
E\left[I_{A}\right]=\int_{0}^{t_{d}} E\left[\ddot{u}^{g}(t)\right] d t=\int_{0}^{t_{d}} f^{2}(t) E\left[\ddot{x}^{g}(t)\right] d t=\left(\int_{0}^{t_{d}} f^{2}(t) d t\right)\left(\int_{-\infty}^{+\infty} S_{\dot{x} \ddot{x}}^{g}(\omega) d \omega\right)
$$


The above relation for the Kanai-Tajimi spectrum and the box-car modulating function can be determined as [53]:

$$
E\left[I_{A}\right]=\frac{t_{d}\left(1+4 \xi_{g}^{2}\right) \pi \omega_{g} S_{0} f_{0}^{2}}{2 \xi_{g}}
$$

The mean Arias intensity can be obtained based on the Modified Mercalli intensity as [55]:

$\log _{10} E\left[I_{A}\right]=\left\{\begin{array}{lll}-1.17+0.560 I_{M M} & \text { for } & \text { horizontal acceleration component } \\ -1.99+0.597 I_{M M} & \text { for } & \text { vertical acceleration component }\end{array}\right.$

with units for $I_{A}$ of $\mathrm{cm}^{2} / \mathrm{sec}^{4}$, and $I I I \leq I_{M M} \leq V I I I . f_{0}$ corresponding to a specific Modified Mercalli intensity can be evaluated using Eqs. 42 and 43. Considering $I_{M M}=V I I I$, then $t_{d}=15.7 \mathrm{sec}$ and $f_{0}=0.016$ for the horizontal component, and $t_{d}=18.94 \mathrm{sec}$ and $f_{0}=0.011$ for the rocking component.

\subsection{Response evaluation}

The effects of the parameters $N_{g}, \beta, \lambda_{x}$ and $\eta$ on the frequency content of the pseudo-static acceleration of straight short-span bridges, with the consideration of the two conditions specified in Section 2, can be investigated using Eq. 18 assuming that $\alpha=0^{\circ}$ as the worst case scenario. In the present evaluation, the relationship between the frequency content of the ground and pseudo-static motions is considered as:

$\left|\ddot{U}_{x}^{p s}(\omega)\right|=\Lambda_{x}(\omega) \sqrt{\left|\ddot{U}_{x}^{g}(\omega)\right|^{2}+h^{2}\left|\ddot{\theta}_{y}^{g}(\omega)^{2}+2 \rho(\omega) h\right| \ddot{U}_{x}^{g}(\omega)|| \ddot{\theta}_{y}^{g}(\omega) \mid}$

where $\rho(\omega)$ is the cross-correlation between the rocking and horizontal ground motions. In utilizing Eq. $44, \rho(\omega)$ is set equal to zero because the rocking component is correlated to the vertical component and, therefore, it should be minimally correlated to the horizontal motions. It is obvious that considering $\rho(\omega)$ $\equiv 1$ (perfect correlation) will unrealistically increase the contribution of the rocking component to the pseudo-static motions. In the subsequent text and figures, the dependency of $\Lambda_{x}(\omega)$ on frequency $(\omega)$ is omitted for convenience.

Fig. 4 presents the variation of $\Lambda_{x}$ versus $\eta$ for $V_{S}=300 \mathrm{~m} / \mathrm{sec}, \beta=90^{\circ}$ (horizontal wave propagation), two values of $\lambda_{x}(0.00$ and 0.45$)$, and $N_{g}=2,3,4$, and 5. It can be seen that $\Lambda_{x}$ decreases with increasing $\eta$, and this reduction is more pronounced for higher values of $\eta$. Furthermore, it can be seen that the rate of the reduction of the pseudo-static acceleration decreases with increasing number of bridge supports. This trend may imply that increase in the number of the supports of a multiple-support structure (or decrease of its span lengths) reduces the effect of the DSGM on the pseudo-static response. It should also be noted that the reduction of $\Lambda_{x}$ becomes very significant when only the time delay of seismic waves is 
considered in the SVSGM formulation $\left(\lambda_{x}=0.00\right.$ in part (a)) as compared to the case when the effects of both loss of coherency $\left(\lambda_{x}=0.45\right)$ and wave propagation $(\beta)$ are incorporated in the SVSGM model (part (b)). In fact, when $\lambda_{x}$ increases (Fig. 4(b)), the destructive interaction of $\lambda_{x}$ and $\beta$ may cause only small variation in $\Lambda_{x}$ as compared to the case when $\lambda_{x}=0$. In the case of $N_{g}=2$ in Fig. 4(b), because of the destructive interaction of the loss of coherency and the wave propagation, the decrease in $\Lambda_{x}$ is even less than all other cases at higher values of $\eta$. Considering Eq. 18, the destructive interaction occurs when $\pi / 2<\left(\ln / N_{g}-1\right) \sin \beta<3 \pi / 2$. Thus, it may be concluded that, for a relatively flat site with soft soil conditions and simple geometrical and material characteristics of its soil layers above the bedrock, the incoherency effects may be conservatively ignored in the estimation of the pseudo-static motions of the multiple-support structures. However, it should be kept in mind that the incoherency effects may become pronounced due to the local scattering generated by topographic and geologic irregularities at a site, even with soft soil conditions.

Fig. 5 presents the variation of $\Lambda_{x}$ versus $\eta$ for $V_{S}=300 \mathrm{~m} / \mathrm{sec}, N_{g}=3$ and variable $\lambda_{x}$ and $\beta$. The figure indicates that the increase of $\lambda_{x}$ causes the reduction of the DSGM effect for $\beta=90^{\circ}$ and $\beta=60^{\circ}$. In contrast, the increase of $\lambda_{x}$ increases the effect of the DSGM for $\beta=0^{\circ}$ and $\beta=30^{\circ}$. Thus, it may be concluded that, for almost horizontally propagating waves $\left(\beta\right.$ close to $90^{\circ}$ ), the increase of the incoherency tends to decrease the effects of the wave propagation. It can also be seen that $\Lambda_{\mathrm{x}}$ decreases with increasing $\eta$, and this reduction is more pronounced for higher values of $\eta$.

In the following, the effect of the non-stationary, multi-support, multi-component excitations on the longitudinal response of a typical four-span bridge, with the consideration of the two conditions specified in Section 2, is investigated using the concept of the MTVRS (Eq. 33). The bridge is supported by three piers $\left(N_{g}=3\right)$, and roller supports are considered at the two ends of its superstructure (Fig. 1). The distance from the first to the third pier is assumed to be equal to $60 \mathrm{~m}(\mathrm{~d}=60 \mathrm{~m})$.

Fig. 6 shows the variation of the $\operatorname{MTVRS}^{u}$ and $\tau_{S V}^{1}$ (Eqs. 32 and 33) versus the structural period for $V_{S}=$ $300 \mathrm{~m} / \mathrm{sec}, \lambda_{x}=0.3, \xi=0.05$ and variable $\beta$. Both uniform and non-uniform excitation scenarios of the horizontal ground motions are considered for the structural loading of the bridge in order to assess the DSGM effect on the MTVRS. The effect of the rocking component on the bridge response is omitted in this case. As can be seen in Fig. 6(a), the MTVRS ${ }^{u}$ retains its highest value for the uniform excitation, i.e. no loss of coherency $\left(\lambda_{x}=0\right)$ and vertically propagating waves $\left(\beta=0^{\circ}\right)$. Additionally, the MTVRS ${ }^{u}$ due to non-uniform excitation decreases with the increase of the incident angle of seismic waves. Fig. 6(b) shows that the $\tau_{S V}^{1}$, at its minimum, drops approximately $28 \%$. The decrease occurs for structural periods shorter than the predominant excitation period of the horizontal acceleration component, which from 
Table 3 is $T_{g}=0.83 \mathrm{sec}$. Hence, DSGMs have a beneficial effect on the seismic input of the bridge. It can also be seen that, past $T_{g}$, the DSGM effect on the MTVRS decreases with the increase of the period of the bridge and tends to fairly constant values.

Fig. 7 shows the variation of the MTVRS ${ }_{\text {nonuniform }}^{u, \theta}$ and $\tau_{S V}^{2}$ (Eqs. 32 and 33) versus the structural period for $h=20 \mathrm{~m}, V_{S}=300 \mathrm{~m} / \mathrm{sec}, \lambda_{x}=0.3, \xi=0.05$ and variable $\beta$. It is recalled that $\tau_{S V}^{2}$ reflects the effect of the combined action of the RSGM and DSGM on the MTVRS in comparison to the case where neither the RSGM nor the DSGM are considered in the bridge excitation. Fig. 7(a) shows that, for a fixed $\lambda_{x}$, the $\operatorname{MTVRS}_{\text {nonuniform }}^{u, \theta}$ decreases with increasing incident angle of the seismic waves. Fig. 7(b) illustrates that the rocking component reduces the maximum contribution of the DSGM to the bridge response (from $28 \%$ to $24 \%$ ) and may even slightly amplify it (at a maximum value around $2 \%$ ) for structural periods shorter than the predominant period of the rocking acceleration component, which from Table 3 is $T_{g}^{\theta}=$ 0.57 sec. Fig. 7 indicates that, overall, the consideration of the effect of the SVSGMs decreases the earthquake input energy and, thus, this phenomenon may have a beneficial effect on the earthquake response of bridges.

Fig. 8 presents the variation of $\tau_{S V}^{3}$ (Eq. 33) versus the structural period for $V_{S}=300 \mathrm{~m} / \mathrm{sec}, \lambda_{x}=0.3$ and $\xi=0.05$, and variable $h$ and $\beta . \tau_{S V}^{3}$ reflects the effect of the combined action of the RSGM and DSGM on the MTVRS in comparison to the case when only DSGM is considered in the bridge excitation. As can be seen from this figure, the effect of the rocking component of the seismic excitation of bridges with $h \leq 10 \mathrm{~m}$ or $T>0.8 \mathrm{sec}$ is negligible in comparison to the DSGM effect. In contrast, for tall bridges with $T$ $<0.8 \mathrm{sec}$, the rocking component can not only reduce the beneficial effect of the DSGM on the seismic loading of such structures, but can also significantly increase the absolute input energy, especially for $\beta=$ $60^{\circ}$. Therefore, it may be deduced that the consideration of the RSGM in the seismic loading tends to decrease the beneficial effects of the DSGM on the bridge response. Fig. 8 also indicates that $\tau_{S V}^{3}$ increases with increasing incident angle of the seismic waves, with the exception of $\beta=90^{\circ}$. This trend may indicate that the RSGM effect on the MTVRS depends more on the variation of the incident angle as compared to the DSGM effect. The exception of $\beta=90^{\circ}$ may be justified due to the destructive interaction of the loss of coherency and incident angle of the input motions on the system response as shown in Fig. 5. This observation can be justified considering the higher frequency content of the SDF of the rocking component in comparison to the horizontal component (Table 3). 
Fig. 9 presents the variation of $\tau_{S V}^{1}, \tau_{S V}^{2}$ and $\tau_{S V}^{3}$ (Eq. 33) versus the structural period for $h=20 m, V_{S}=$ $300 \mathrm{~m} / \mathrm{sec}, \beta=60^{\circ}$ and variable $\lambda_{x}$. The comparison of $\tau_{S V}^{1}$ with $\tau_{S V}^{2}$ and $\tau_{S V}^{3}$ indicates that the variation of $\lambda_{x}$ has a higher influence on the contribution of the rocking component to the MTVRS in comparison to the DSGM. Considering the variation of $\tau_{S V}^{2}$ in Fig. 9, it can also be seen, that $\lambda_{x}$ plays a reverse role on the contribution of the DSGM and the RSGM to the MTVRS before and after the predominant period of the horizontal acceleration component. For bridges with periods shorter than the predominant period of the horizontal acceleration component, the increase of $\lambda_{x}$ decreases their effect for $\tau_{S V}^{2}$, whereas the opposite occurs at higher periods. Considering the variation of $\tau_{S V}^{3}$, it appears that the increase of $\lambda_{x}$ increases the contribution of the RSGM to the MTVRS, and the maximum contribution occurs at the predominant period of the rocking acceleration component.

Fig. 10 presents the variation of $\tau_{S V}^{1}, \tau_{S V}^{2}$ and $\tau_{S V}^{3}$ (Eq. 33) versus the structural period for $h=20 m, \lambda_{x}=$ $0.45, \beta=60^{\circ}$ and variable $V_{S}$. Considering the variation of $\tau_{S V}^{1}$ and $\tau_{S V}^{3}$, it can be seen that the increase of the shear wave velocity decreases the effects of both the DSGM and the RSGM. For $V_{S}=200 \mathrm{~m} / \mathrm{sec}$, the maximum contribution of the SVSGM to the MTVRS for $\tau_{S V}^{1}$ and $\tau_{S V}^{3}$ is $34 \%$ and $42 \%$, respectively. For $V_{S}=400 \mathrm{~m} / \mathrm{sec}$, this maximum contribution for $\tau_{S V}^{1}$ and $\tau_{S V}^{3}$ decreases to $17 \%$ and $15 \%$, respectively. The variation of the $\tau_{S V}^{2}$ illustrates the significance of the frequency content of the SVSGM in the input energy of bridges. As can be seen from the figure, the consideration of the SVSGM in the bridge excitation leads to a slight increase of the absolute input energy if the fundamental frequency of the bridge is just below the predominant frequency of the rocking component (Table 3). On the other hand, the consideration of the SVSGM in the bridge excitation leads to the decrease of the absolute input energy if the fundamental frequency of the bridge is lower than the predominant frequency of the horizontal component. These effects are more predominant for the lower values of $V_{S}$. Finally, it should be noted that even though the DSGM may have a beneficial effect on the absolute input energy of bridges (variation of $\tau_{S V}^{1}$ in Figs. 6, 9 and 10), the RSGM may dramatically decrease this effect and increase the absolute input energy of bridges (variation of $\tau_{S V}^{2}$ in Figs. 7, 9 and 10) depending on the variation of the SVSGM parameters, i.e. $\beta, \lambda_{x}$, and $V_{S}$.

\section{Conclusions}


In this paper, a new procedure for the investigation of the effect of the non-stationary SVSGMs on the behavior of multiple-support structures has been proposed, and its application to straight, short-span bridges has been investigated. In this approach, the seismic loading due to both the DSGM and RSGM has been considered, and a technique for the estimation of the mean total velocity response spectrum (MTVRS) has been presented using random vibration and input energy methods. The main advantage of the proposed approach is its ability to easily and efficiently estimate the effects of the combined action of the rotational and translational components of the seismic excitation on the mean absolute input energy of ordinary bridges, which are the most common bridge structural systems. For modeling the SDF of RSGMs, a low-pass filter is also introduced to modify the Kanai-Tajimi spectrum in order to prevent the root-mean-square of the rocking spectrum from becoming infinite. The main finding of the present study is that, if the SVSGM effects are going to be considered in the earthquake excitation of bridges, both the DSGM and RSGM should be taken into account in seismic design. Other conclusions that may be drawn from the numerical results are summarized in the following:

- The consideration of the loss of coherency increases considerably the amplitude of the SDF of the rocking component, and leads to more critical rotational excitation of the structures. Hence, the combined action of both loss of coherency and time delay should be taken into account in the simulation of the RSGMs.

- The increase of the number of piers (or decrease of the span lengths) of an ordinary bridge with stiff superstructure tends to decrease the effects of the DSGM on its pseudo-static response.

- For incident angles of $\sin ^{-1}\left(\pi\left(N_{g}-1\right) / 2 l \eta\right)<\beta<\sin ^{-1}\left(3 \pi\left(N_{g}-1\right) / 2 l \eta\right)$, the destructive interaction between the time delay and loss of coherency decreases the DSGM effects on the pseudo-static acceleration of regular multiple-support structures. This reduction becomes maximum for almost horizontally propagating seismic waves. Thus, for soft soil conditions, it may be conservative to neglect the incoherency effects for the evaluation of the pseudo-static acceleration.

- The destructive interaction between the time delay and loss of coherency may considerably decrease the RSGMs effects on the bridge response because of their higher frequency content as compared to the translational motions.

- The maximum effect of the SVSGMs on the MTVRS occurs for stiff bridges and depends mostly on the predominant frequency of the seismic excitation. The predominant frequency of the horizontal component mostly controls the maximum DSGMs contribution to the MTVRS, and the absolute input energy of the bridge considerably decreases if its fundamental frequency is close to the predominant frequency of the horizontal component. Therefore, the SVSGM may considerably decrease the detrimental effects of the resonant frequency on the bridge response. 
- For short-span bridges, the DSGM decreases the earthquake input energy (variation of $\tau_{S V}^{1}$ ) and, hence, its effect may be considered beneficial on the bridge response. However, the RSGM decreases the beneficial effect of the DSGM on the input energy (variation of $\tau_{S V}^{2}$ ).

- The increase of the loss of coherency (or decrease of the shear wave velocity) increases the RSGM

effects on the MTVRS (variation of $\tau_{S V}^{3}$ ). The maximum increase in the input energy due to the rocking component occurs for tall bridges.

In closing, it should be noted that SSI can also lead to both DSGM and RSGM excitations, but this phenomenon was omitted herein to purely underline the SVSGM contribution to the seismic response of multiple-support structures. Further research is necessary to obtain insight into the importance of the DSGM and RSGM in structural analysis due to the combined action of the SVSGM and SSI effects.

\section{Acknowledgments}

This study was supported in part by the National Science Foundation under Grants No. CMMI-0900179 and CMMI-1129396. Any opinions, findings, and conclusions or recommendations expressed in this material are those of the authors and do not necessarily reflect the views of the National Science Foundation.

\section{Appendix A}

The appendix provides the mathematical expressions for $M_{j}(t, \omega)$ and $N_{j}(t, \omega)$ for a box-car type modulating function. $M_{j}(t, \omega)$ and $N_{j}(t, \omega)$ can be written as:

$\left.M_{j}(t, \omega)=\operatorname{Re}\left[M_{j}(t, \omega)\right]+i \operatorname{Im} \mid M_{j}(t, \omega)\right]$

$N_{j}(t, \omega)=\operatorname{Re}\left[N_{j}(t, \omega)\right]+i \operatorname{Im}\left[N_{j}(t, \omega)\right]$

where $\operatorname{Re}[$.$] and \operatorname{Im}[$.$] refer to the real and imaginary parts of the function under bracket, respectively. The$ real and imaginary quantities of $M_{j}(t, \omega)$ can be obtained as: 


$$
\begin{aligned}
&\left.\operatorname{Re}\left[M_{j}(t, \omega)\right]=-\frac{f_{0}}{2}\right]\left[\frac{\left(\left(-\xi_{j} \omega_{j}\right) / \omega_{d j}\right)\left(\omega_{d j}+\omega\right)+\left(\xi_{j} \omega_{j}\right)}{\left(\omega_{d j}+\omega\right)^{2}+\left(\xi_{j} \omega_{j}\right)^{2}}+\frac{\left(\left(-\xi_{j} \omega_{j}\right) / \omega_{d j}\right)\left(\omega_{d j}-\omega\right)+\left(\xi_{j} \omega_{j}\right)}{\left(\omega_{d j}-\omega\right)^{2}+\left(\xi_{j} \omega_{j}\right)^{2}}\right]-e^{\left(-\xi_{j} \omega_{j}\right) t} \times \\
& {\left.\left[\frac{\left[\left(\left(-\xi_{j} \omega_{j}\right) / \omega_{d j}\right)\left(\omega_{d j}+\omega\right)+\left(\xi_{j} \omega_{j}\right)\right] \cos \left(\left(\omega_{d j}+\omega\right) t\right)-\left[\left(\omega_{d j}+\omega\right)+\left(\left(\xi_{j} \omega_{j}\right)^{2} / \omega_{d j}\right)\right] \sin \left(\left(\omega_{d j}+\omega\right) t\right)}{\left(\omega_{d j}+\omega\right)^{2}+\left(\xi_{j} \omega_{j}\right)^{2}}\right]\right] } \\
&\left.\left.+\frac{\left.\left[\left(\left(-\xi_{j} \omega_{j}\right) / \omega_{d j}\right)\left(\omega_{d j}-\omega\right)+\left(\xi_{j} \omega_{j}\right)\right] \cos \left(\left(\omega_{d j}-\omega\right) t\right)-\left[\left(\omega_{d j}-\omega\right)+\left(\left(\xi_{j} \omega_{j}\right)^{2} / \omega_{d j}\right)\right] \sin \left(\left(\omega_{d j}-\omega\right) t\right)\right]}{\left(\omega_{d j}-\omega\right)^{2}+\left(\xi_{j} \omega_{j}\right)^{2}}\right]\right] \\
& \operatorname{Im}\left[M_{j}(t, \omega)\right]=-\frac{f_{0}}{2}\left[\left[\frac{\left(\omega_{d j}+\omega\right)-\left(\left(\xi_{j} \omega_{j}\right)^{2} / \omega_{d j}\right)}{\left(\omega_{d j}+\omega\right)^{2}+\left(\xi_{j} \omega_{j}\right)^{2}}-\frac{\left(\omega_{d j}-\omega\right)+\left(\left(\xi_{j} \omega_{j}\right)^{2} / \omega_{d j}\right)}{\left(\omega_{d j}-\omega\right)^{2}+\left(\xi_{j} \omega_{j}\right)^{2}}\right]-e^{\left(-\xi_{j} \omega_{j}\right) t} \times\right. \\
& {\left.\left[\frac{\left[\left(\omega_{d j}+\omega\right)+\left(\left(\xi_{j} \omega_{j}\right)^{2} / \omega_{d j}\right)\right] \cos \left(\left(\omega_{d j}+\omega\right) t\right)+\left[\left(\left(-\xi_{j} \omega_{j}\right) / \omega_{d j}\right)\left(\omega_{d j}+\omega\right)+\left(\xi_{j} \omega_{j}\right)\right] \sin \left(\left(\omega_{d j}+\omega\right) t\right)}{\left(\omega_{d j}+\omega\right)^{2}+\left(\xi_{j} \omega_{j}\right)^{2}}\right]\right] } \\
&\left.\left.+\frac{\left[-\left(\omega_{d j}-\omega\right)-\left(\left(\xi_{j} \omega_{j}\right)^{2} / \omega_{d j}\right)\right] \cos \left(\left(\omega_{d j}-\omega\right) t\right)+\left[-\left(\left(-\xi_{j} \omega_{j}\right) / \omega_{d j}\right)\left(\omega_{d j}-\omega\right)+\left(\xi_{j} \omega_{j}\right)\right] \sin \left(\left(\omega_{d j}-\omega\right) t\right)}{\left(\xi_{j} \omega_{j}\right)^{2}}\right]\right]
\end{aligned}
$$

and $N_{j}(t, \omega)$ can be also obtained as:

$$
\begin{aligned}
& \operatorname{Re}\left[N_{j}(t, \omega)\right]=-\frac{f_{0}^{2}}{2}\left[\left[\left(\frac{\left(\left(-\xi_{j} \omega_{j}\right) / \omega_{d j}\right)\left(\omega_{d j}+\omega\right)+\left(\xi_{j} \omega_{j}\right)}{\left(\omega_{d j}+\omega\right)^{2}+\left(\xi_{j} \omega_{j}\right)^{2}}+\frac{\left(\left(-\xi_{j} \omega_{j}\right) / \omega_{d j}\right)\left(\omega_{d j}-\omega\right)+\left(\xi_{j} \omega_{j}\right)}{\left(\omega_{d j}-\omega\right)^{2}+\left(\xi_{j} \omega_{j}\right)^{2}}\right] t\right]-e^{\left(-\xi_{j} \omega_{j}\right) t} \times\right. \\
& {\left[\frac{\left[\left(\left(-\xi_{j} \omega_{j}\right) / \omega_{d j}\right)\left(\omega_{d j}+\omega\right)+\left(\xi_{j} \omega_{j}\right)\right]\left(\left(-\xi_{j} \omega_{j}\right) \cos \left(\left(\omega_{d j}+\omega\right) t\right)+\left(\omega_{d j}+\omega\right) \sin \left(\left(\omega_{d j}+\omega\right) t\right)+\left(\xi_{j} \omega_{j}\right) e^{\left(\xi_{j} \omega_{j}\right) t}\right)}{\left(\left(\omega_{d j}+\omega\right)^{2}+\left(\xi_{j} \omega_{j}\right)^{2}\right)^{2}}\right.} \\
& -\frac{\left[\left(\omega_{d j}+\omega\right)+\left(\left(\xi_{j} \omega_{j}\right)^{2} / \omega_{d j}\right)\right]\left(\left(-\xi_{j} \omega_{j}\right) \sin \left(\left(\omega_{d j}+\omega\right) t\right)-\left(\omega_{d j}+\omega\right) \cos \left(\left(\omega_{d j}+\omega\right) t\right)+\left(\omega_{d j}+\omega\right) e^{\left(\xi_{j} \omega_{j}\right) t}\right)}{\left(\left(\omega_{d j}+\omega\right)^{2}+\left(\xi_{j} \omega_{j}\right)^{2}\right)^{2}} \\
& +\frac{\left[\left(\left(-\xi_{j} \omega_{j}\right) / \omega_{d j}\right)\left(\omega_{d j}-\omega\right)+\left(\xi_{j} \omega_{j}\right)\right]\left(\left(-\xi_{j} \omega_{j}\right) \cos \left(\left(\omega_{d j}-\omega\right) t\right)+\left(\omega_{d j}-\omega\right) \sin \left(\left(\omega_{d j}-\omega\right) t\right)+\left(\xi_{j} \omega_{j}\right) e^{\left(\xi_{j} \omega_{j}\right) t}\right)}{\left(\left(\omega_{d j}-\omega\right)^{2}+\left(\xi_{j} \omega_{j}\right)^{2}\right)^{2}} \\
& \left.\left.-\frac{\left[\left(\omega_{d j}-\omega\right)+\left(\left(\xi_{j} \omega_{j}\right)^{2} / \omega_{d j}\right)\right]\left(\left(-\xi_{j} \omega_{j}\right) \sin \left(\left(\omega_{d j}-\omega\right) t\right)-\left(\omega_{d j}-\omega\right) \cos \left(\left(\omega_{d j}-\omega\right) t\right)+\left(\omega_{d j}-\omega\right) e^{\left(\xi_{j} \omega_{j}\right) t}\right)}{\left(\left(\omega_{d j}-\omega\right)^{2}+\left(\xi_{j} \omega_{j}\right)^{2}\right)^{2}}\right]\right] \\
& \operatorname{Im}\left[N_{j}(t, \omega)\right]=-\frac{f_{0}^{2}}{2}\left[\left[\left(\frac{\left(\omega_{d j}+\omega\right)+\left(\left(\xi_{j} \omega_{j}\right)^{2} / \omega_{d j}\right)}{\left(\omega_{d j}+\omega\right)^{2}+\left(\xi_{j} \omega_{j}\right)^{2}}-\frac{\left(\omega_{d j}-\omega\right)+\left(\left(\xi_{j} \omega_{j}\right)^{2} / \omega_{d j}\right)}{\left(\omega_{d j}-\omega\right)^{2}+\left(\xi_{j} \omega_{j}\right)^{2}}\right) t\right]-e^{\left(-\xi_{j} \omega_{j}\right) t} \times\right. \\
& {\left[\frac{\left[\left(\omega_{d j}+\omega\right)+\left(\left(\xi_{j} \omega_{j}\right)^{2} / \omega_{d j}\right)\right]\left(\left(-\xi_{j} \omega_{j}\right) \cos \left(\left(\omega_{d j}+\omega\right) t\right)+\left(\omega_{d j}+\omega\right) \sin \left(\left(\omega_{d j}+\omega\right) t\right)+\left(\xi_{j} \omega_{j}\right) e^{\left(\xi_{j} \omega_{j}\right) t}\right)}{\left(\left(\omega_{d j}+\omega\right)^{2}+\left(\xi_{j} \omega_{j}\right)^{2}\right)^{2}}\right.} \\
& +\frac{\left[\left(\left(-\xi_{j} \omega_{j}\right) / \omega_{d j}\right)\left(\omega_{d j}+\omega\right)+\left(\xi_{j} \omega_{j}\right)\right]\left(\left(-\xi_{j} \omega_{j}\right) \sin \left(\left(\omega_{d j}+\omega\right) t\right)-\left(\omega_{d j}+\omega\right) \cos \left(\left(\omega_{d j}+\omega\right) t\right)+\left(\omega_{d j}+\omega\right) e^{\left(\xi_{j} \omega_{j}\right) t}\right)}{\left(\left(\omega_{d j}+\omega\right)^{2}+\left(\xi_{j} \omega_{j}\right)^{2}\right)^{2}} \\
& +\frac{\left[-\left(\omega_{d j}-\omega\right)-\left(\left(\xi_{j} \omega_{j}\right)^{2} / \omega_{d j}\right)\right)\left(\left(-\xi_{j} \omega_{j}\right) \cos \left(\left(\omega_{d j}-\omega\right) t\right)+\left(\omega_{d j}-\omega\right) \sin \left(\left(\omega_{d j}-\omega\right) t\right)+\left(\xi_{j} \omega_{j}\right) e^{\left(\xi_{j} \omega_{j}\right) t}\right)}{\left(\left(\omega_{d j}-\omega\right)^{2}+\left(\xi_{j} \omega_{j}\right)^{2}\right)^{2}} \\
& \left.\left.+\frac{\left[-\left(\left(-\xi_{j} \omega_{j}\right) / \omega_{d j}\right)\left(\omega_{d j}-\omega\right)+\left(-\xi_{j} \omega_{j}\right)\right]\left(\left(-\xi_{j} \omega_{j}\right) \sin \left(\left(\omega_{d j}-\omega\right) t\right)-\left(\omega_{d j}-\omega\right) \cos \left(\left(\omega_{d j}-\omega\right) t\right)+\left(\omega_{d j}-\omega\right) e^{\left(\xi_{j} \omega_{j}\right) t}\right)}{\left(\left(\omega_{d j}-\omega\right)^{2}+\left(\xi_{j} \omega_{j}\right)^{2}\right)^{2}}\right]\right]
\end{aligned}
$$




\section{References}

1. M. D. Trifunac, A note on rotational components of earthquake motions on ground surface for incident body waves, Soil Dynamics and Earthquake Engineering, Vol. 1 (1982) 11-19.

2. D. Basu, A. S. Whittaker, M. C. Constantinou, Extracting rotational components of earthquake ground motion using data recorded at multiple stations, Earthquake Engineering and Structural Dynamics, 42 (2013) 451-463.

3. J. E. Luco, D. A. Sotiropouls, Local characterization of free-field ground motion and effects of wave passage, Bulletin of the Seismological Society of America, Vol. 70 (1980) 2229-2244.

4. A. Rutenberg, A. C. Heidebrecht, Response spectra for torsion, rocking and rigid foundations, Earthquake Engineering and Structural Dynamics, Vol. 13 (1985) 543-557.

5. M. Ghafory-Ashtiany, M. P. Singh, Structural response for six correlated earthquake components, Earthquake Engineering and Structural Dynamics, Vol. 14 (1986) 103-119.

6. Z. Zembaty, G. Boffi, Effect of rotational seismic ground motion on dynamic response of slender towers, European Earthquake Engineering, Vol. 1 (1994) 3-11.

7. E. Kalkan, V. Grazer, Coupled tilt and translational ground motion response spectra, Journal of Earthquake Engineering, Vol. 133 (2007) 609-619.

8. M. R. Falamarz-Sheikhabadi, M. Ghafory-Ashtiany, Spatial seismic excitations of long structures, Sixth International Conference of Seismology and Earthquake Engineering (SEE6), 2011 Tehran, IRAN.

9. M. Ghafory-Ashtiany, M. R. Falamarz-Sheikhabadi, Evaluation influence of rotational components on the behavior of structures, Report International Institute of Earthquake Engineering and Seismology, IIEES, in Farsi (2010).

10. M. R. Falamarz-Sheikhabadi, M. Ghafory-Ashtiany, Approximate formulas for rotational effects in earthquake engineering, Journal of Seismology, Vol. 16 (2012) 815-827.

11. M. I. Todorovska, M. D. Trifunac, A note on excitation of long structures by ground waves, Journal of Engineering Mechanics division (ASCE), Vol. 116 (1990) 952-964.

12. A. Der Kiureghian, A. Neuenhofer, Response spectrum method for multi-support seismic excitations. Earthquake Engineering and Structural Dynamics, Vol. 21 (1992) 715-740.

13. Z. Zembaty, A. Rutenberg, Spatial response spectra and site amplification, Engineering Structures, Vol. 24 (2002) 1485-1496.

14. A. Zerva, Spatial variation of seismic ground motions: modeling and engineering applications, CRC Press, Taylor \& Francis Group (2009).

15. M. Takeo, Ground rotational motions recorded in near-source region of earthquakes, Geophysical Research Letters, Vol. 25 (1998) 789792.

16. B. S. Huang, Ground rotational motions of the 1999 Chi-Chi, Taiwan earthquake as inferred from dense array observations, Geophysical Research Letters, Vol. 30 (2003) 40 1-4.

17. M. D. Trifunac, The role of strong motion rotations in the response of structures near earthquake faults, Soil Dynamics and Earthquake Engineering, Vol. 29 (2009) 382-393.

18. M. R. Falamarz-Sheikhabadi, M. S. Nazari, Behavior of multi-support structures subjected to rotational earthquake components, $15^{\text {th }}$ World Conference on Earthquake Engineering, 2012 Lisboan, Portugal.

19. C. M. Uang, V. V. Bertero, Evaluation of seismic energy in structures, Earthquake Engineering and Structural Dynamics, Vol. 19 (1990) $77-90$.

20. M. Ordaz, B. Heuerta, E. Reinoso, Exact computation of input-energy spectra from Fourier amplitude spectra, Earthquake Engineering and Structural Dynamics, Vol. 32 (2003) 597-605.

21. R. Ruiz, J. Penzien, Probabilistic study of the behaviour of structures during earthquakes, Report EERC-69-03, Earthquake Engineering Research Center, University of California at Berkeley, CA (1969).

22. Z. A. Der, R. H. Shumay and A. C. Lees, Frequency domain coherent processing of regional seismic signals at small arrays, Bulletin Seismic Society of America, Vol. 70 (326-388) 1988.

23. A. K. Chopra, J. T. Wang, Earthquake response of arch dams to spatially varying ground motion, Earthquake Engineering and Structural Dynamics, Vol. 39 (2010) 887-906.

24. M. D. Trifunac, M. I. Todorovska, Response spectra for differential motion of columns, Earthquake Engineering and Structural Dynamics, Vol. 26 (1997) 251-268.

25. Eurocode 8, Design of structures for earthquake resistance, Part 2: Bridges, EN 1998-2, (2005).

26. A. G. Sextos, A. J. Kappos, Evaluation of seismic response of bridges under asynchronous excitation and comparisons with Eurocode 8-2 provisions, Bulletin of Earthquake Engineering, Vol. 7 (2009) 519-545.

27. E. Heredia-Zavoni, F. Barranco, Torsion in symmetric structures due to ground-motion spatial variation, Journal of Engineering Mechanics, Vol. 122 (1996) 834-843.

28. S. Liao, A. Zerva, Physically-compliant, conditionally simulated spatially variable ground motions for performance-based design, Earthquake Engineering and Structural Dynamics, Vol. 35 (2006) 891-919.

29. L. Lou, A. Zerva, Effects of spatially variable ground motions on the seismic response of a skewed, multi-span, RC highway bridge, Soil Dynamics and Earthquake Engineering, Vol. 25 (2005) 729-740.

30. M. R. Falamarz-Sheikhabadi, Simplified relations for the application of rotational components to seismic design codes, Engineering Structures, Vol. 59 (2014) 141-152.

31. M. R. Falamarz-Sheikhabadi, M. Ghafory-Ashtiany, Rotational components in structural loading, Soil Dynamics and Earthquake Engineering, Vol. 75 (2015) 220-233.

32. S. Maleki, Deck modeling for seismic analysis of skewed slab-girder bridges, Engineering Structures, Vol. 24 (2002) 1315-1326.

33. M. J. N. Priestley, F. Seible, G. M. Calvi, Seismic design and retrofit of bridges, John Wiley \& Sons, Inc., (1996).

34. M. Bouchon, K. Aki, Strain, tilt, and rotation associated with strong ground motion in the vicinity of earthquake faults, Bulletin of the Seismological Society of America, Vol. 72 (1982) 1717-1738

35. Z. Zembaty, A. Castellani, G. Boffi, Spectral analysis of the rotational component of seismic ground motion, Probabilistic Engineering Mechanics, Vol. 8 (1993) 5-14. 
36. K. Kanai, Semi-empirical formula for the seismic characteristic of the ground, Bulletin of Earthquake Research Institute, Tokyo, Vol. 35 (1957) 309-325.

37. H. Tajimi, A statistical method of determining the maximum response of a building structure during an earthquake, In Proceedings of $2^{\text {nd }}$ World Conference on Earthquake Engineering, Tokyo, Vol. 2 (1960) 781-798.

38. G. W. Housner, Behavior of structures during earthquakes, Journal of the Engineering Mechanics Division, ASCE, Vol. 85 (1959) $109-129$.

39. N. Tzanetos, A. S. Elnashai, F. H. Hamdan and S. Antoniou, Inelastic dynamic response of RC bridges subjected to spatially nonsynchronous earthquake motion, Advances in Structural Engineering, Vol. 3 (2000) 191-214.

40. G. Zanardo., H. Hao., C., Modena, Seismic response of multi-span simply supported bridges to a spatially varying earthquake ground motion, Earthquake Engineering and Structural Dynamics, Vol. 31 (2002) 1325-1345.

41. D. Basu., A. S. Whittaker., M. C. Constantinou., Characterizing rotational components of earthquake ground motion using a surface distribution method and response of sample structures, Engineering Structures, Vol. 99 (2015) 685-707.

42. Eurocode 8, Design provisions for earthquake resistance of structures, European Committee for Standardization, Part 6: Tower, masts, and chimneys, EN1998-6, (2005).

43. W. J. Sun, A. Kareem, Response of MDOF systems to nonstationary random excitation, Engineering Structures, Vol. 11 (1989) 83-91.

44. M. Barbato, J. P. Conte, Spectral characteristics of non-stationary random processes: Theory and application to linear structural models, Engineering Mechanics, Vol. 23 (2008) 416-426.

45. B. Zhou, X. Han, S. K. Tan, A simplified computational method for random seismic responses of a jacket platform, Ocean Engineering, Vol. 82 (2014) 85-90.

46. S. Ates, Seismic behavior of isolated multi-span continuous bridge to nonstationary random seismic excitation, Nonlinear dynamics, Vol. 67 (2012) 263-282.

47. R. S. Harichandran, A. Hawwari, B. N. Sweidan, Response of long-span bridges to spatially varying ground motion, Journal of Structural Engineering, Vol. 122 (1996) 476-484.

48. I. D. Gupta, M. D. Trifunac, A note on the nonstationary of seismic response of structures, Engineering Structures, Vol. 23 (2000) 15671577.

49. X. L. Jin, Z. L. Huang, A. Y. T. Leung, Nonstationary seismic responses of structure with nonlinear stiffness subject to modulated KanaiTajimi excitation, Earthquake Engineering and Structural Dynamics, Vol. 41 (2012) 197-210.

50. Y. A. Chakraborty, B. Basu, Nonstationary response analysis of long span bridges under spatially varying differential support motions using continuous wavelet transformation, Journal of Engineering Mechanics, Vol. 134 (2008) 155-162.

51. I. Takewaki, Bound of earthquake input energy, Journal of Structural Engineering, Vol. 130 (2004) 1289-1297.

52. M. B. Priestley, Power spectral analysis of nonstationary random process, Journal of Sound Vibration, Vol. 6 (1967) 86-97.

53. Z. Zembaty, A note on non-stationary stochastic response and strong ground motion duration, Earthquake Engineering and Structural dynamics, Vol. 16 (1988) 1189-1200.

54. R. S. Jangid, T. K. Datta, Evaluation of the methods for response analysis under non-stationary excitation, Shock and Vibration, Vol. 6 (1999) 285-297.

55. M. D. Trifunac, A. G. Brady, A study on the duration of strong earthquake ground motion, Bulletin of the Seismological Society of America, Vol. 65 (1975) 581-626.

56. S. P. Lai, Seismic response of a 4-span bridge system subjected to multiple-support ground excitation, Proceedings of $4^{\text {th }}$ Canadian Conference on Earthquake Engineering, Vancouver (1983).

57. A. Rutenberg, A. C. Heidebrecht, Approximate spectral multiple support seismic analysis: traveling wave approach, Proceedings of the institution of Civil Engineers, Part 2, Research and Theory, (1988) 223-236.

58. Jalali, R. S., Trifunac, M. D., Response spectra for near-source, differential and rotational strong ground motion, Bulletin of the Seismological Society of America, Vol. 99 (2009) 1404-1415.

59. Arias, A., A measure of earthquake intensity, in R. J. Hansen, ed., Seismic for Nuclear Power Plants, MIT Press, Cambridge, Massachusetts, (1970) 438-483.

60. N. A. Abrahamson, J. F. Schneider, J. C. Stepp, Spatial coherency of shear waves from the Lotung, Taiwan large-scale seismic test, Structural Safety, Vol. 10 (1991) 145-162. 


\section{Tables}

Table 1. Values of $b_{N g}$ and $a_{l}$ for straight short-span bridges with different support number.

Table 2. Parameters of single and double filtered Kanai-Tajimi spectrum.

Table 3. Predominant periods of spectral density function of the horizontal and rocking components.

\section{Figures}

Figure 1. Model of multiple-support structure.

Figure 2. Schematic diagram of the seismic wave propagation from the hypocenter to the site.

Figure 3. Spectral density functions of translational (left side) and rocking (right side) acceleration components generated using single and double filtered Kanai-Tajimi spectrum.

Figure 4. Variation of $\Lambda_{x}$ versus $\eta=\omega d / V_{S}$ for $V_{S}=300 \mathrm{~m} / \mathrm{sec}, \beta=90^{\circ}$ and two values of $\lambda_{x}$.

Figure 5. Variation of $\Lambda_{x}$ versus $\eta=\omega d / V_{S}$ for $N_{g}=3, V_{S}=300 \mathrm{~m} / \mathrm{sec}$ and variable $\lambda_{x}$ and $\beta$.

Figure 6. Variation of $\mathrm{MTVRS}^{u}$ and $\tau_{S V}^{1}$ versus the structural period for $V_{S}=300 \mathrm{~m} / \mathrm{sec}, \lambda_{x}=0.3, \xi=0.05$ and variable $\beta$.

Figure 7. Variation of MTVRS ${ }_{\text {nonuniform }}^{u, \theta}$ and $\tau_{S V}^{2}$ versus the structural period for $h=20 \mathrm{~m}, V_{S}=300 \mathrm{~m} / \mathrm{sec}, \lambda_{x}=0.3$, $\xi=0.05$ and variable $\beta$.

Figure 8. Variation of $\tau_{S V}^{3}$ versus the structural period for $V_{S}=300 \mathrm{~m} / \mathrm{sec}, \lambda_{x}=0.3$ and variable $h$ and $\beta$.

Figure 9. Variation of $\tau_{S V}^{1}, \tau_{S V}^{2}$ and $\tau_{S V}^{3}$ versus the structural period for $h=20 \mathrm{~m}, V_{S}=300 \mathrm{~m} / \mathrm{sec}, \beta=60^{\circ}$ and variable $\lambda_{x}$.

Figure 10. Variation of $\tau_{S V}^{1}, \tau_{S V}^{2}$ and $\tau_{S V}^{3}$ versus the structural period for $h=20 m, \lambda_{x}=0.45, \beta=60^{\circ}$ and variable $V_{S}$. 
Table 1

Values of $b_{N g}$ and $a_{l}$ for straight short-span bridges with different support number.

\begin{tabular}{|c|c|c|c|c|c|c|c|c|}
\hline $\boldsymbol{N}_{g}$ & $\boldsymbol{b}_{N g}$ & $\boldsymbol{a}_{1}$ & $\boldsymbol{a}_{2}$ & $\boldsymbol{a}_{3}$ & $\boldsymbol{a}_{4}$ & $\boldsymbol{a}_{5}$ & $\boldsymbol{a}_{6}$ & $\boldsymbol{a}_{7}$ \\
\hline$\underline{\mathbf{2}}$ & $1 / 2$ & $-1 / 4$ & 0 & 0 & 0 & 0 & 0 & 0 \\
\hline$\underline{\mathbf{3}}$ & $1 / 2$ & 0 & $-1 / 4$ & 0 & 0 & 0 & 0 & 0 \\
\hline$\underline{\mathbf{4}}$ & $5 / 9$ & $5 / 36$ & $-1 / 6$ & $-1 / 4$ & 0 & 0 & 0 & 0 \\
\hline$\underline{\mathbf{5}}$ & $5 / 8$ & $1 / 4$ & $-1 / 16$ & $-1 / 4$ & $-1 / 4$ & 0 & 0 & 0 \\
\hline$\underline{\mathbf{6}}$ & $7 / 10$ & $7 / 20$ & $1 / 25$ & $-19 / 100$ & $-3 / 10$ & $-1 / 4$ & 0 & 0 \\
\hline$\underline{\mathbf{7}}$ & $7 / 9$ & $4 / 9$ & $5 / 36$ & $-1 / 9$ & $-5 / 18$ & $-1 / 3$ & $-1 / 4$ & 0 \\
\hline$\underline{\mathbf{8}}$ & $6 / 7$ & $15 / 28$ & $23 / 98$ & $-5 / 196$ & $-11 / 49$ & $-67 / 196$ & $-5 / 14$ & $-1 / 4$ \\
\hline
\end{tabular}

Table 2

Parameters of single and double filtered Kanai-Tajimi spectrum.

\begin{tabular}{|c|c|c|c|c|c|c|}
\hline $\mathrm{SDF}$ & $\xi_{f}$ & $\omega_{f}(\mathrm{rad} / \mathrm{sec})$ & $\xi_{g}$ & $\omega_{g}(\mathrm{rad} / \mathrm{sec})$ & $S_{0}^{\text {Single Filter }}\left(\mathrm{m}^{2} / \mathrm{sec}^{4}\right)$ & $S_{0}^{\text {Double Filter }}\left(\mathrm{m}^{2} / \mathrm{sec}^{4}\right)$ \\
\hline Vertical component & 0.6 & 1 & 0.4 & 10 & 0.31 & 0.4 \\
\hline $\begin{array}{c}\text { Horizontal } \\
\text { component }\end{array}$ & 0.6 & 0.75 & 0.3 & 7.5 & 0.78 & 1 \\
\hline
\end{tabular}

Table 3

Predominant periods of spectral density function of the horizontal and rocking components.

\begin{tabular}{|c|c|c|c|}
\hline Component & Acceleration SDF $(\mathrm{sec})$ & Velocity SDF $(\mathrm{sec})$ & Displacement SDF $(\mathrm{sec})$ \\
\hline Horizontal & 0.83 & 7.69 & 10.00 \\
\hline Rocking & 0.57 & 0.72 & 6.67 \\
\hline
\end{tabular}




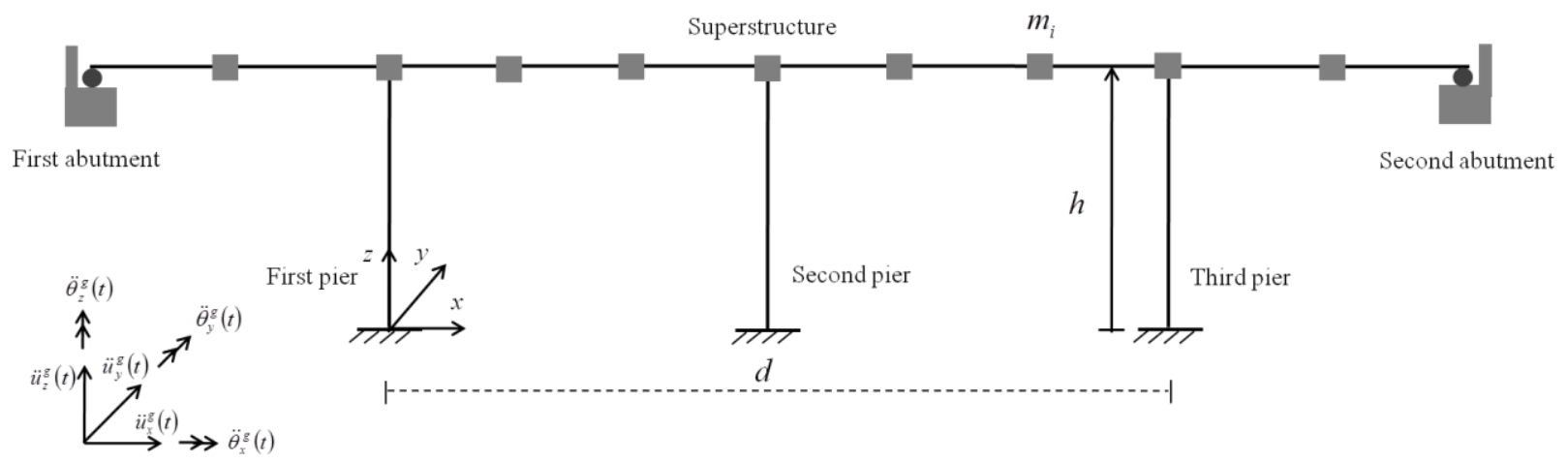

Figure 1. Model of multiple-support structure.

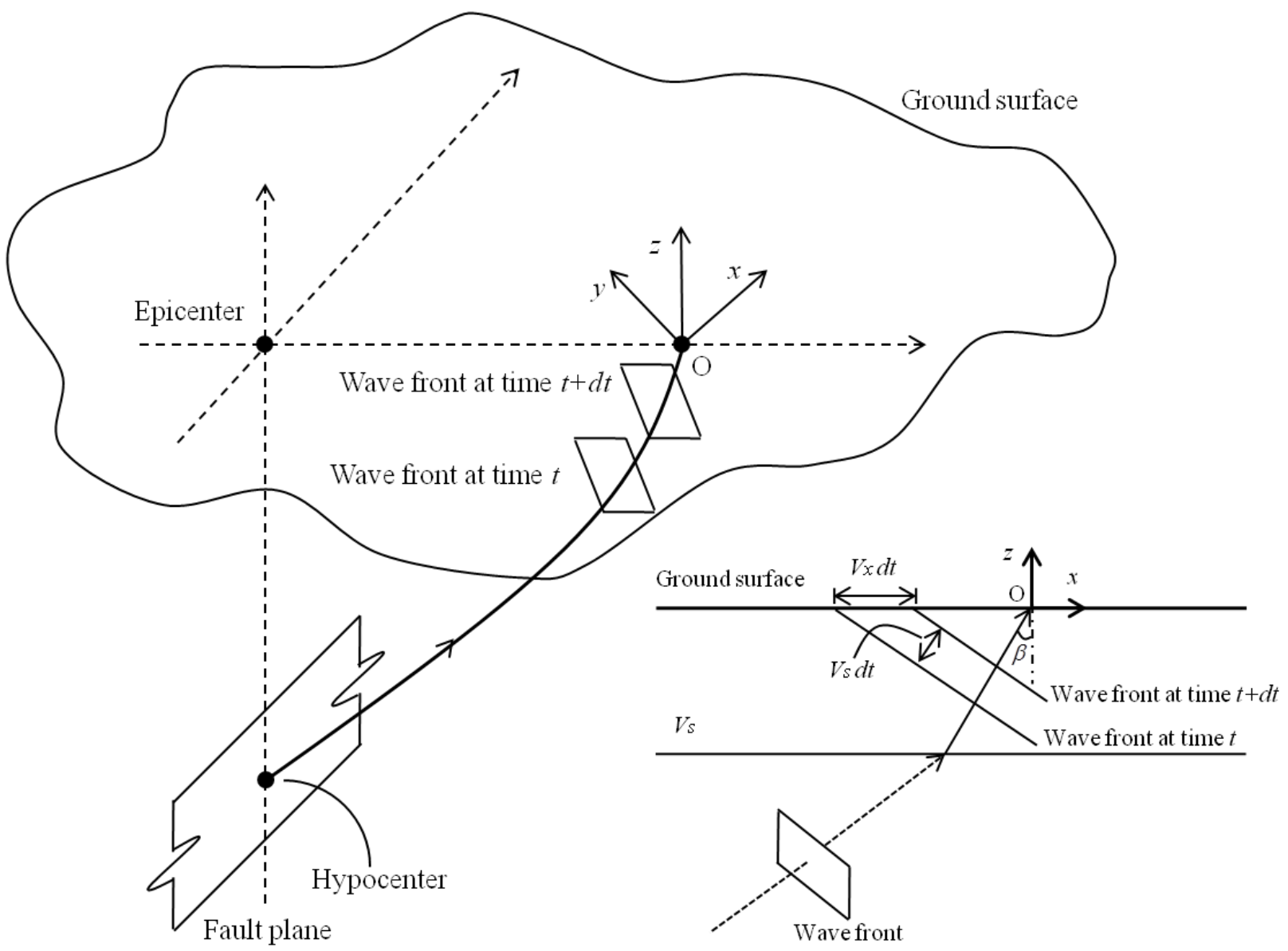

Figure 2. Schematic diagram of the seismic wave propagation from the hypocenter to the site. 
Single filtered Kanai-Tajimi spectrum

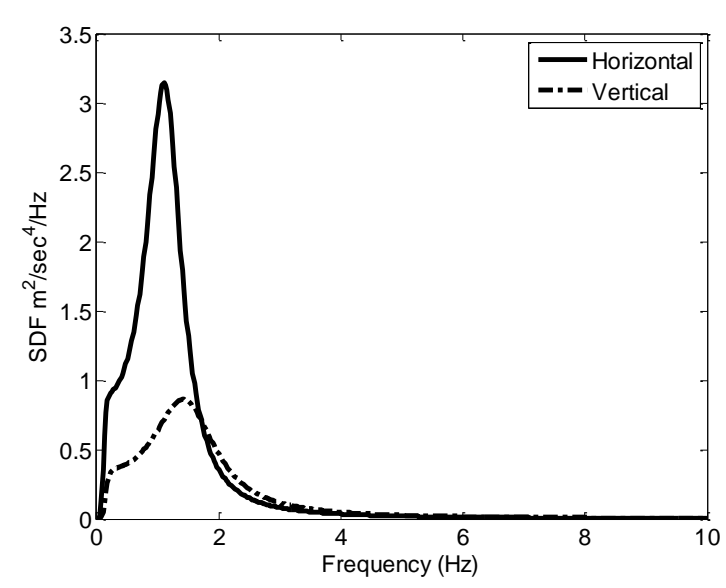

(a) translational components

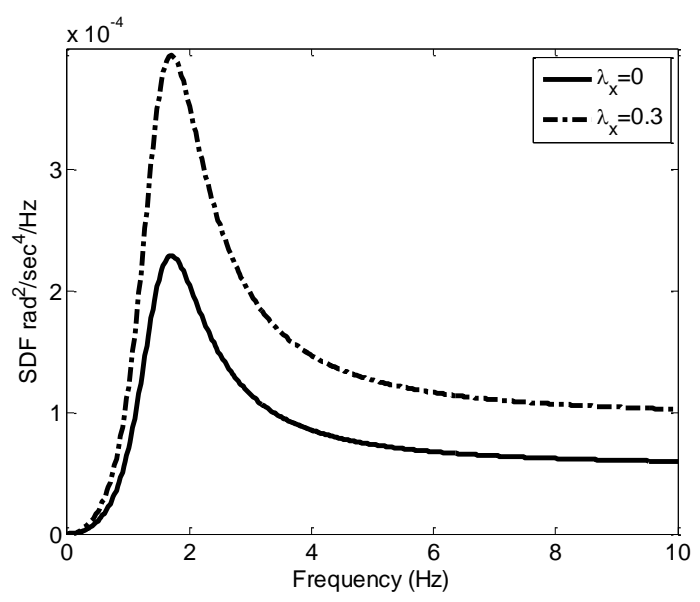

(b) rocking component

Double filtered Kanai-Tajimi spectrum

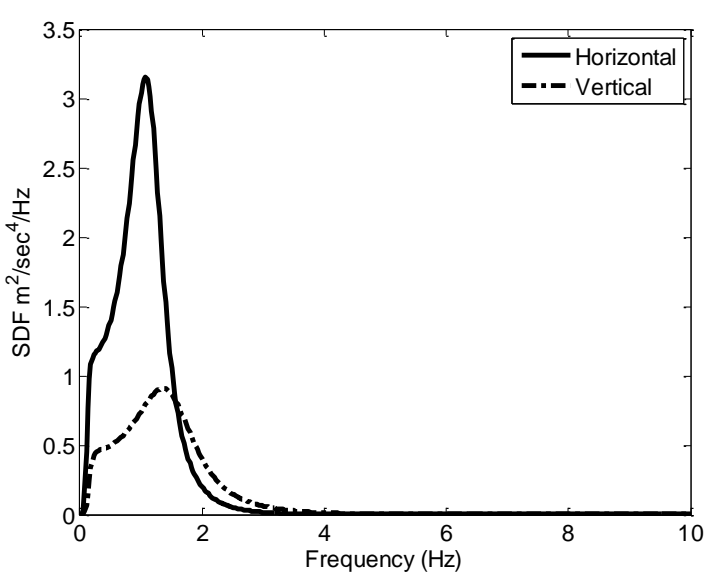

(c) translational components

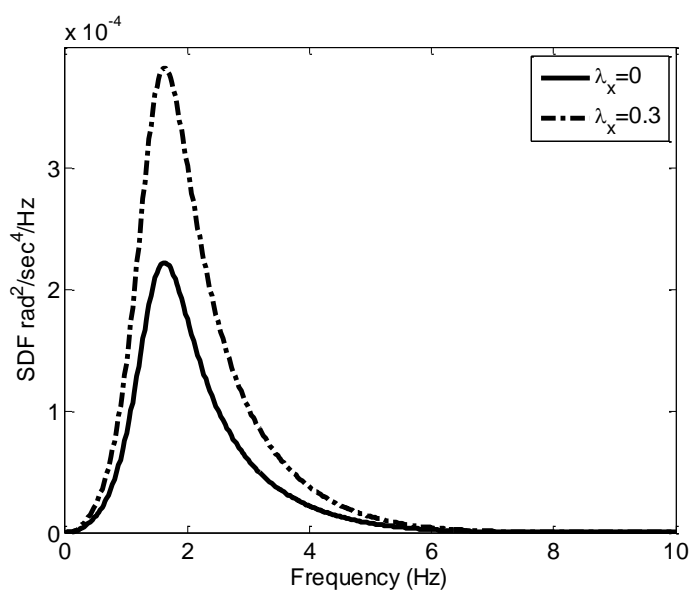

(d) rocking component

Figure 3. Spectral density functions of translational (left side) and rocking (right side) acceleration components generated using single and double filtered Kanai-Tajimi spectrum. 


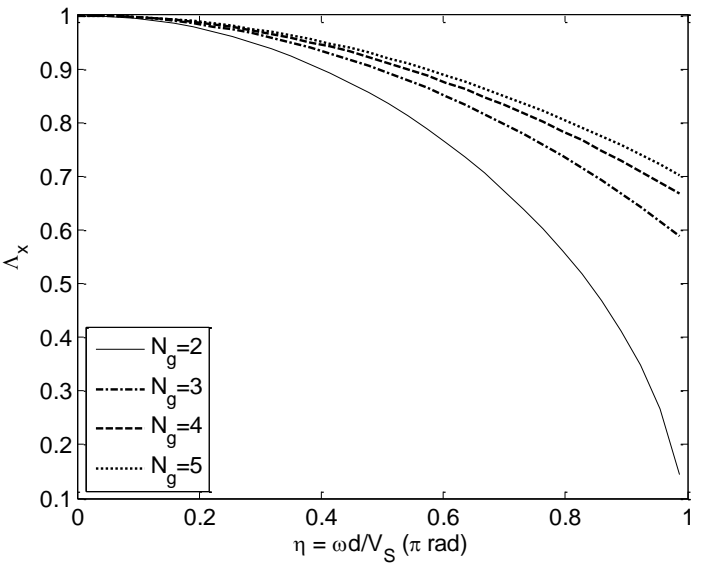

(a) $\lambda_{x}=0.00$

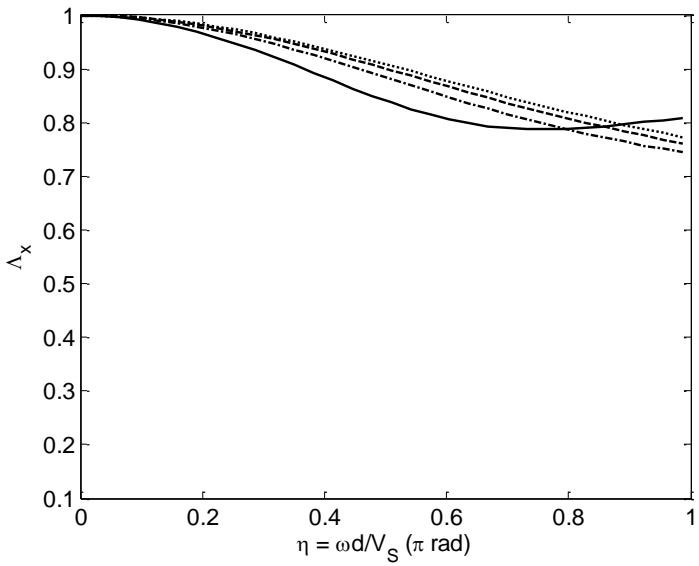

(b) $\lambda_{x}=0.45$

Figure 4. Variation of $\Lambda_{x}$ versus $\eta=\omega d / V_{S}$ for $V_{S}=300 \mathrm{~m} / \mathrm{sec}, \beta=90^{\circ}$ and two values of $\lambda_{x}$. 


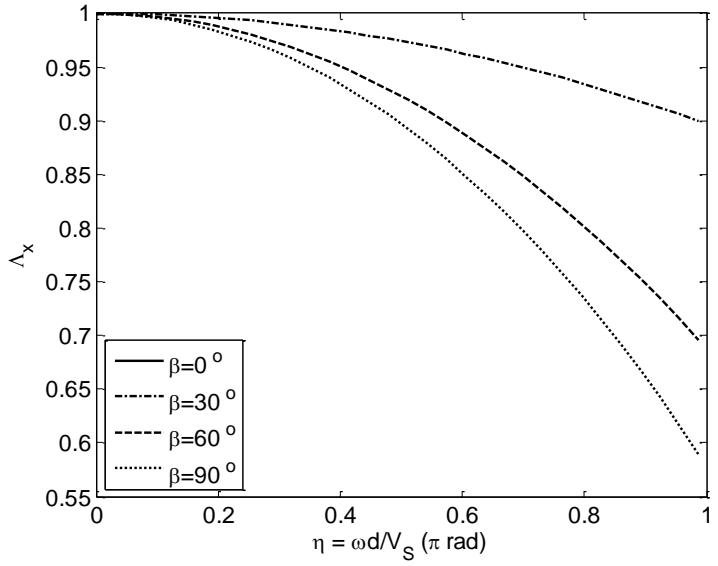

(a) $\lambda_{x}=0.00$

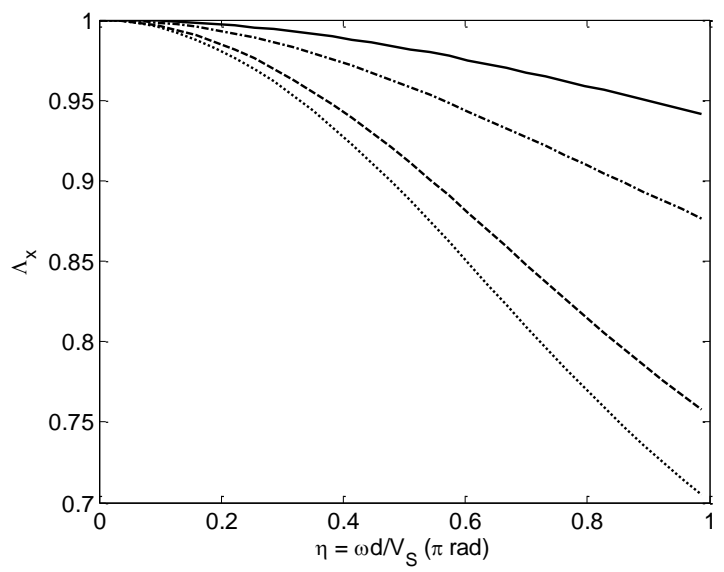

(c) $\lambda_{x}=0.30$

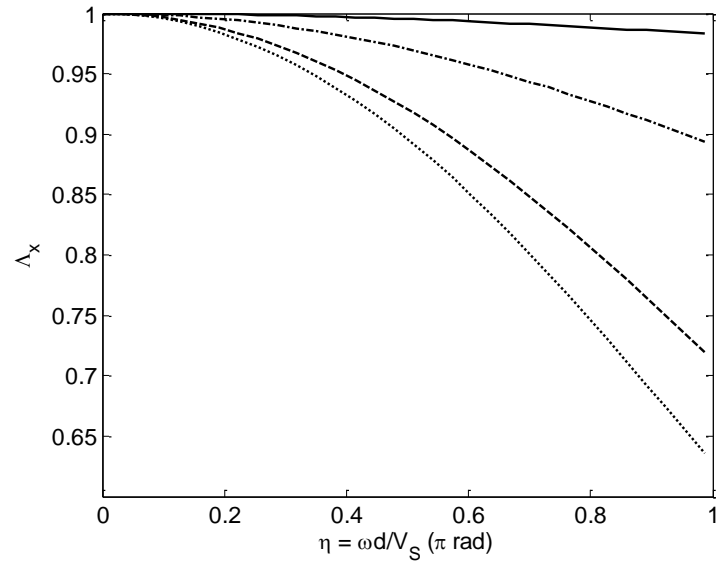

(b) $\lambda_{x}=0.15$

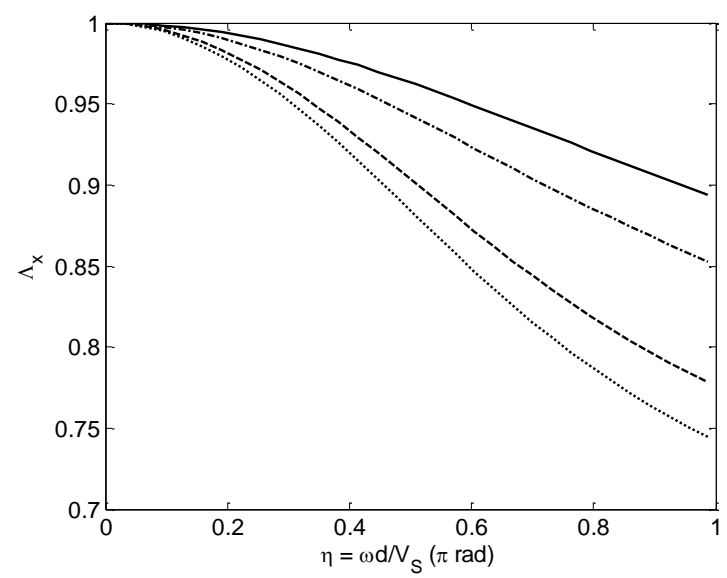

(d) $\lambda_{x}=0.45$

Figure 5. Variation of $\Lambda_{x}$ versus $\eta=\omega d / V_{S}$ for $N_{g}=3, V_{S}=300 \mathrm{~m} / \mathrm{sec}$ and variable $\lambda_{x}$ and $\beta$. 


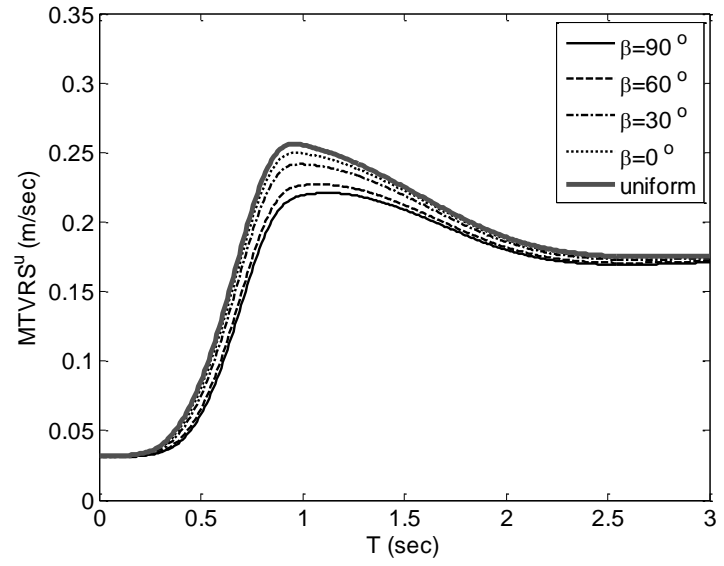

(a)

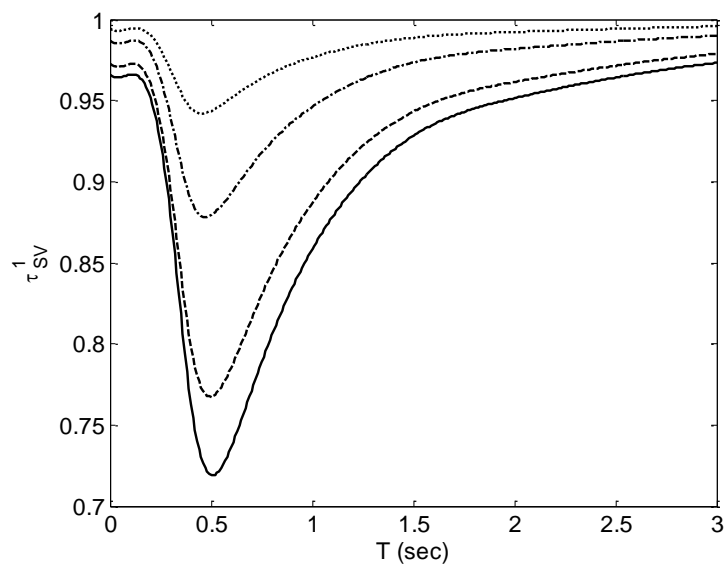

(b)

Figure 6. Variation of $\mathrm{MTVRS}^{u}$ and $\tau_{S V}^{1}$ versus the structural period for $V_{S}=300 \mathrm{~m} / \mathrm{sec}, \lambda_{x}=0.3, \xi=0.05$ and variable $\beta$.

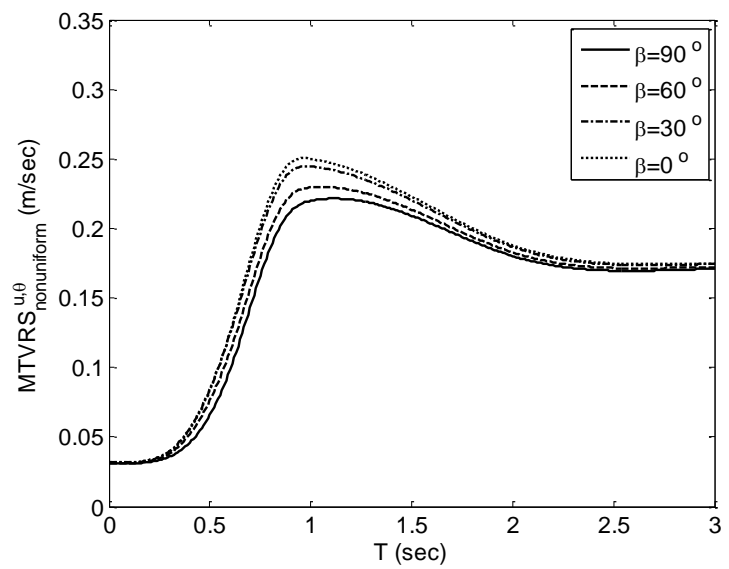

(a)

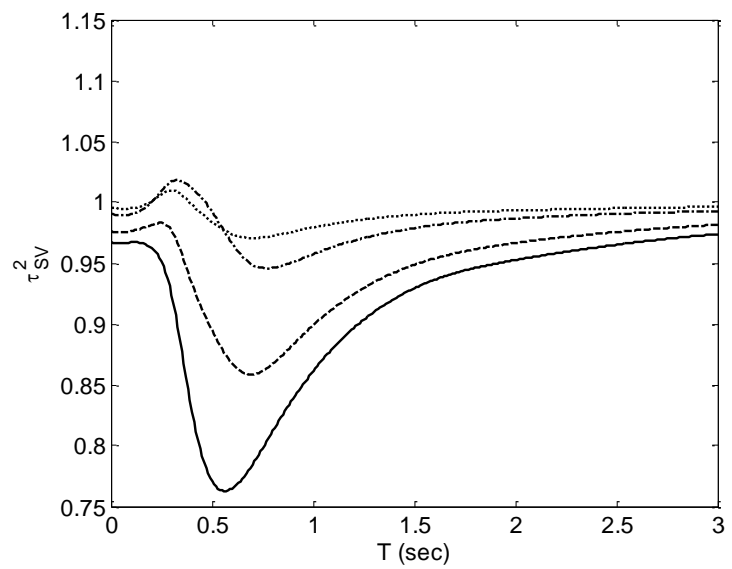

(b)

Figure 7. Variation of MTVRS $\underset{\text { nonuniform }}{u, \theta}$ and $\tau_{S V}^{2}$ versus the structural period for $h=20 \mathrm{~m}, V_{S}=300 \mathrm{~m} / \mathrm{sec}, \lambda_{x}=0.3$, $\xi=0.05$ and variable $\beta$. 


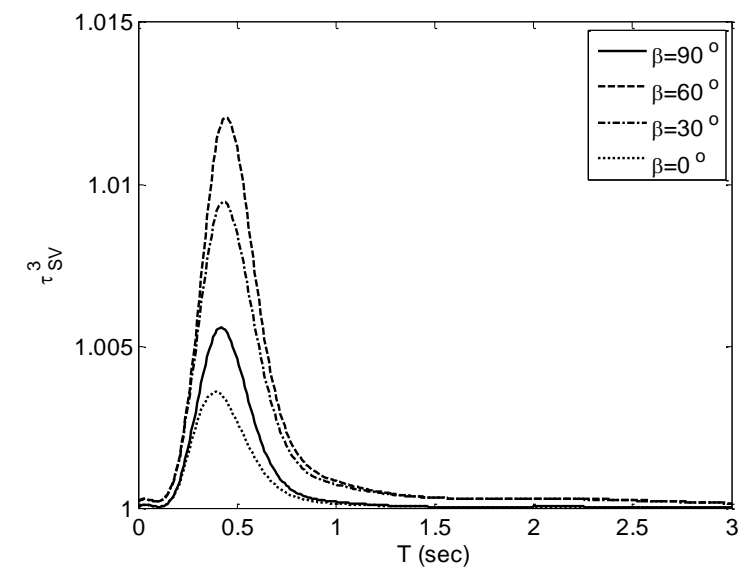

(a) $h=5 m$

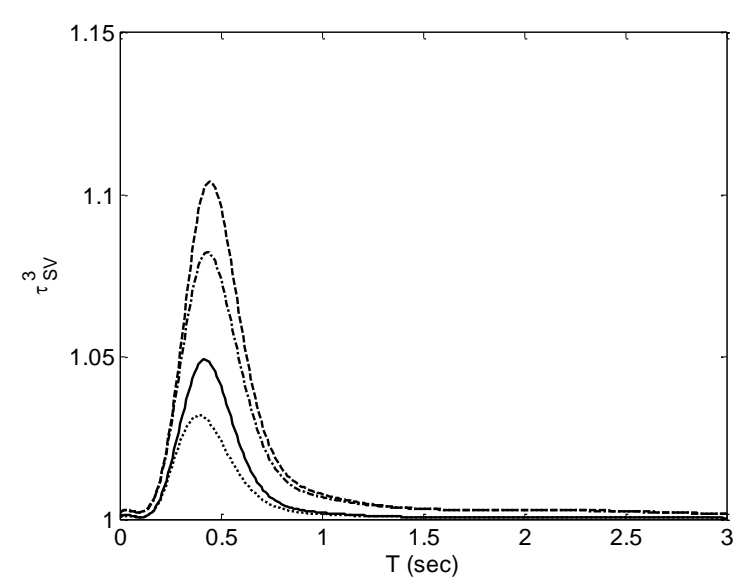

(c) $h=15 \mathrm{~m}$

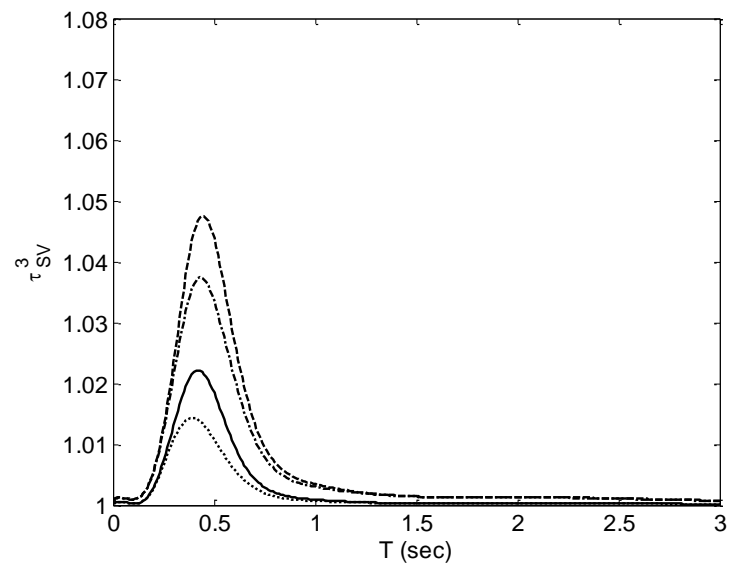

(b) $h=10 m$

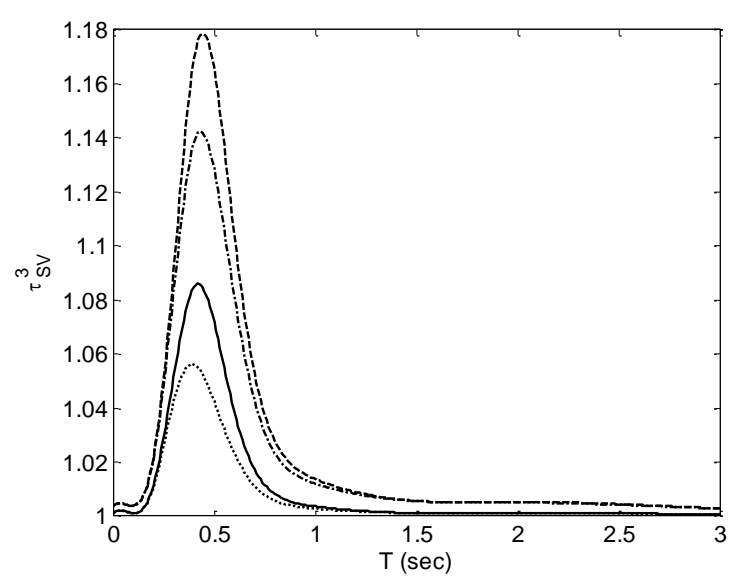

(d) $h=20 \mathrm{~m}$

Figure 8. Variation of $\tau_{S V}^{3}$ versus the structural period for $V_{S}=300 \mathrm{~m} / \mathrm{sec}, \lambda_{x}=0.3$ and variable $h$ and $\beta$. 

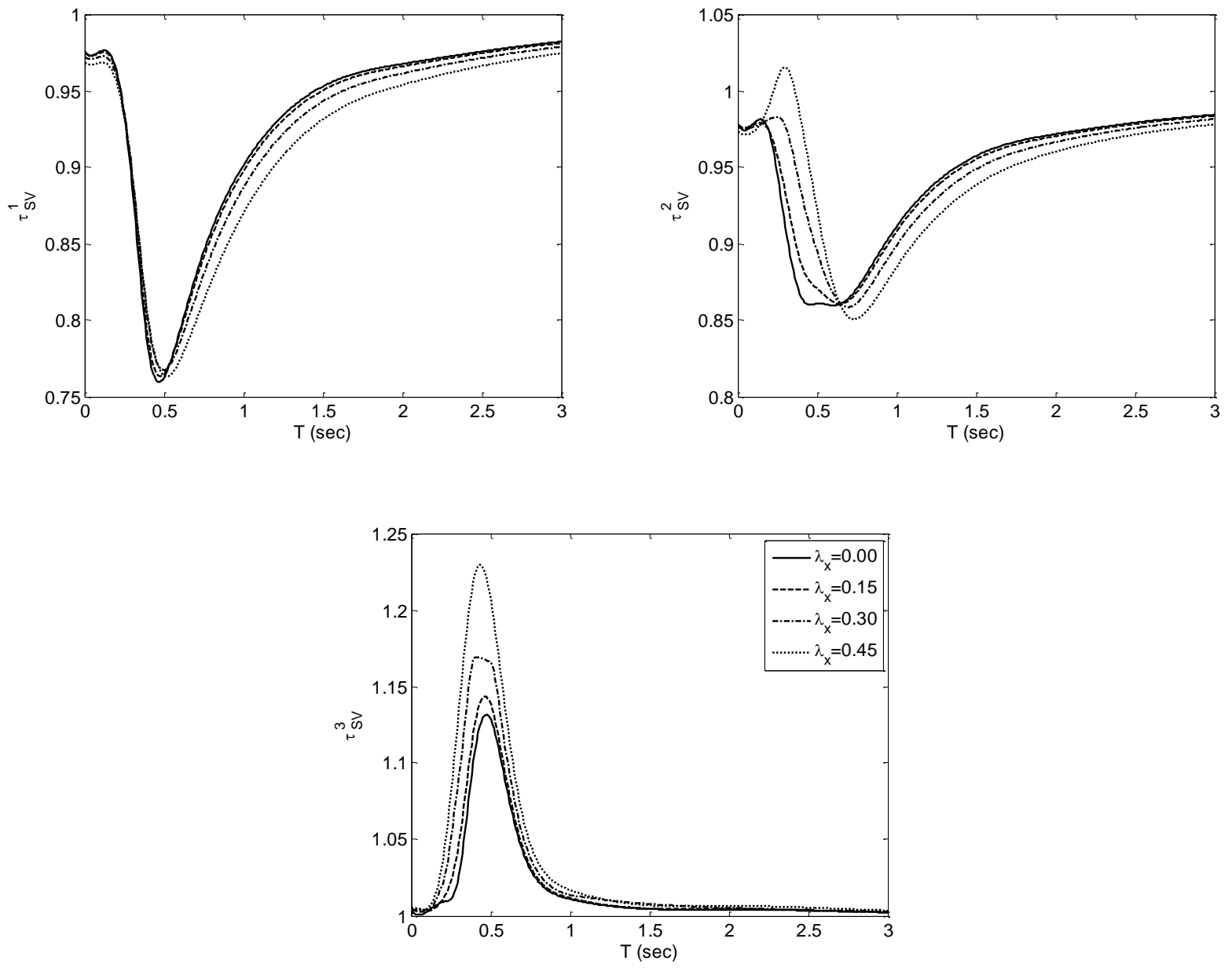

Figure 9. Variation of $\tau_{S V}^{1}, \tau_{S V}^{2}$ and $\tau_{S V}^{3}$ versus the structural period for $h=20 \mathrm{~m}, V_{S}=300 \mathrm{~m} / \mathrm{sec}, \beta=60^{\circ}$ and variable $\lambda_{x}$. 

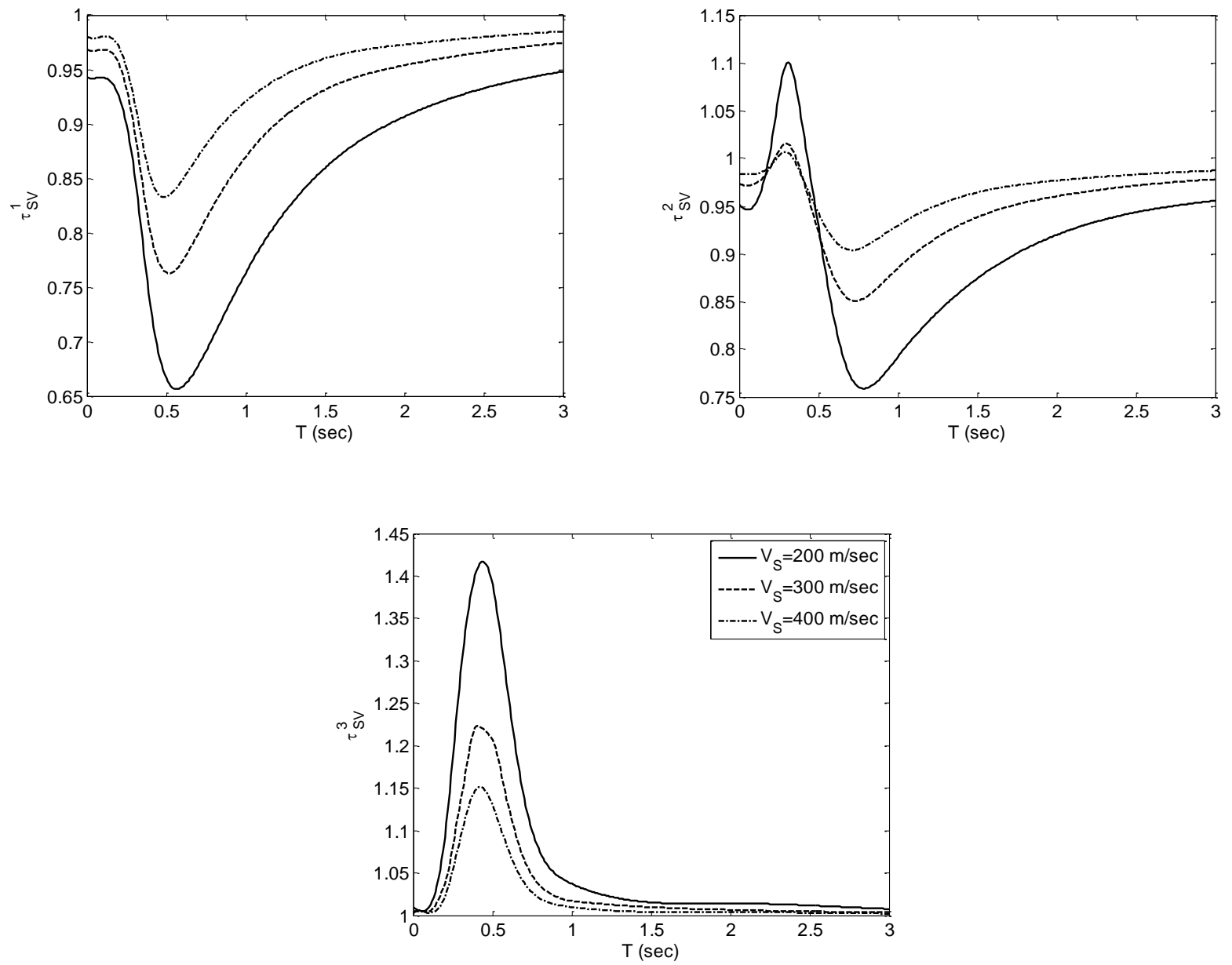

Figure 10. Variation of $\tau_{S V}^{1}, \tau_{S V}^{2}$ and $\tau_{S V}^{3}$ versus the structural period for $h=20 m, \lambda_{x}=0.45, \beta=60^{\circ}$ and variable $V_{S}$. 